\title{
ON SUPERCRITICAL NONLINEAR SCHRÖDINGER EQUATIONS WITH ELLIPSE-SHAPED POTENTIALS
}

\author{
JIANFU YANG \\ Department of Mathematics, Jiangxi Normal University \\ Nanchang, Jiangxi 330022, P. R. China \\ email: Jianfu Yang: jfyang_2000@yahoo.com \\ JINGE YANG* \\ School of Sciences, Nanchang Institute of Technology \\ Nanchang 330099, P. R. China \\ email: Jinge Yang: jgyang2007@yeah.net
}

\begin{abstract}
In this paper, we study the existence and concentration of normalized solutions to the supercritical nonlinear Schrödinger equation

$$
\left\{\begin{array}{l}
-\Delta u+V(x) u=\mu_{q} u+a|u|^{q} u \text { in } \mathbb{R}^{2}, \\
\int_{\mathbb{R}^{2}}|u|^{2} d x=1
\end{array}\right.
$$

where $\mu_{q}$ is the Lagrange multiplier. For ellipse-shaped potentials $V(x)$, we show that for $q>2$ close to 2 , the equation admits an excited solution $u_{q}$, and furthermore, we study the limiting behavior of $u_{q}$ when $q \rightarrow 2_{+}$. Particularly, we describe precisely the blow-up formation of the excited state $u_{q}$.
\end{abstract}

\section{INTRODUCTION}

In this paper, we study the following supercritical Gross-Pitaevskii (GP) type equation

$$
-\Delta u+V(x) u=\mu_{q} u+a|u|^{q} u \quad \text { in } \quad \mathbb{R}^{2},
$$

that is, $q>2$, with the ellipse-shaped potential

$$
V(x)=\left(\sqrt{\frac{x_{1}^{2}}{b_{1}^{2}}+\frac{x_{2}^{2}}{b_{2}^{2}}}-A\right)^{2},
$$

where $b_{1}>b_{2}>0, A>0$ are constants, and $\mu_{q}$ is the Lagrange multiplier.

In the case $q=2$, equation (1.1) stems from the study of Bose-Einstein condensation. It was derived independently by Gross and Pitaevskii, and it is the main theoretical tool for investigating nonuniform dilute Bose gases at low temperatures. Especially, equation (1.1) is called the Gross-Pitaevskii equation.

Key words: Nonlinear elliptic equation, $L^{2}$ supercritical, Constrained minimization, GrossCPitaevskii functional, Ellipse-shaped potential. 
In general, problem (1.1) is associated with critical points of the energy functional

$$
E_{a, q}(u)=\frac{1}{2} \int_{\mathbb{R}^{2}}\left(|\nabla u(x)|^{2}+V(x)|u(x)|^{2}\right) d x-\frac{a}{q+2} \int_{\mathbb{R}^{2}}|u|^{q+2} d x
$$

under the constraint

$$
\int_{\mathbb{R}^{2}}|u(x)|^{2} d x=1
$$

In particular, in the critical or subcritical case, that is, $q=2$ or $0<q<2$, the functional $E_{a, q}$ is bounded below if $0<a<a^{*}$ for some $a^{*}>0$. Hence, the constrained minimization problem

$$
d_{a}(q):=\inf _{u \in \mathcal{H}, \int_{\mathbb{R}^{2}}|u|^{2} d x=1} E_{a, q}(u)
$$

is well defined, where $\mathcal{H}$ is defined by

$$
\mathcal{H}:=\left\{u \in H^{1}\left(\mathbb{R}^{2}\right): \int_{\mathbb{R}^{2}} V(x)|u(x)|^{2} d x<\infty\right\} .
$$

In the critical case $q=2$, for $a>0$ the system of Bose-Einstein condensates collapses if the particle number increases beyond a critical value; see [7, 9, 15, 20] etc. Mathematically, it was proved in [11] that for a non-negative potential $V(x)$ with finite number zero points, there exists a threshold value $a^{*}>0$ such that $d_{a}(2)$ is achieved if $0<a<a^{*}$, and there is no minimizer for $d_{a}(2)$ if $a \geq a^{*}$. The threshold value $a^{*}$ is determined in terms of the solution of the nonlinear scalar field equation

$$
-\Delta u+u=u^{3} \text { in } \mathbb{R}^{2}, \quad u \in H^{1}\left(\mathbb{R}^{2}\right) .
$$

It is known from [16] that problem (1.6) admits a unique positive solution up to translations. Such a solution is radially symmetric and exponentially decaying at infinity, see for instance, [5]. Denote by $Q$ in the sequel the positive solution of (1.6). It was found in [11] that

$$
a^{*}:=\|Q\|_{L^{2}\left(\mathbb{R}^{2}\right)}^{2}
$$

The similar symmetry breaking phenomenon was considered in [13] in the subcritical case, i.e. $0<q<2$, for the functional $E_{a, q}$. When $q$ approaching 2 , the limit behavior of the minimizer of $E_{a, q}$ constrained by (1.4) is described by the unique positive solution $\varphi_{q}$ of the nonlinear scalar field equation

$$
-\Delta u+\frac{2}{q} u=\frac{2}{q} u^{q+1}, \quad q>0, \quad u \in H^{1}\left(\mathbb{R}^{2}\right)
$$

in terms of

$$
a_{q}^{*}=\left\|\varphi_{q}\right\|_{2}^{q}
$$

If the non-negative potential $V(x)$ has infinitely many minima, or particular $V(x)=(|x|-$ $A)^{2}$, it was shown in [12] that the symmetry breaking occurs in the GP minimizers. That is, any non-negative minimizer of $E_{a, 2}$ concentrates at a point on the ring $\left\{x \in \mathbb{R}^{2}:|x|=A\right\}$ as $a \rightarrow a_{-}^{*}$. Such a result was generalized to the case that the potential $V(x)$ is ellipse-shaped in $[10]$. 
The situation becomes different if we turn to the supercritical case $q>2$. Arguments for critical and subcritical cases can not be carried through for the supercritical case, since in the supercritical case the functional $E_{a, q}(u)$ is not bounded below on the manifold

$$
S(1)=\left\{u \in \mathcal{H}: \int_{\mathbb{R}^{2}}|u|^{2} d x=1\right\}
$$

and the minimization problem (1.5) is not well defined.

In this paper, we focus on the supercritical problem (1.1) with ellipse-shaped potentials. Although in this case, there is no minimizer for the minimization problem (1.5), or no ground state solution for (1.1), we can find critical points of $E_{a, q}$ constrained on the manifold $S(1)$. Such a critical point is an excited state solution of (1.1). Actually, for the supercritical case, the functional $E_{a, q}$ has the mountain pass geometry on $S(1)$. This was revealed in $[3,14]$, and developed to be applied to various problems, see [2, 4] etc. We first look for critical points of $E_{a, q}$ on $S(1)$ by the variant mountain pass theorem, then we investigate the asymptotic behavior of the critical points when $q$ tends to 2 .

In the sequel, we denote $|x|_{b}=\sqrt{b_{1}^{-2} x_{1}^{2}+b_{2}^{-2} x_{2}^{2}}$. Choose $a \in\left(0, a^{*}\right)$ and denote

$$
\tau_{q}=\left(\frac{2 a_{q}^{*}}{q a}\right)^{\frac{1}{q-2}}
$$

in the sequel. Our existence results are stated as follows.

Theorem 1.1. Let $0<a<a^{*}$. There exists an $\varepsilon_{0}>0$ such that, for any $q \in\left(2,2+\varepsilon_{0}\right)$, $E_{a, q}(u)$ admits a nonnegative critical point $u_{q}$ at mountain level on $S(1)$.

One may verify that the functional $E_{a, q}(u)$ has the mountain pass geometry on $S(1)$. Then it is standard to show that $E_{a, q}(u)$ has a Palais-Smale $(P S)$ sequence at the mountain pass level. However, such a $(P S)$ sequence may fail to be bounded. In order to bound $(P S)$ sequence, we use a variant mountain pass theorem inspired of [14].

Next, we study the limit behavior of mountain pass point $u_{q}$ as $q \rightarrow 2_{+}$.

Theorem 1.2. Suppose $0<a<a^{*}$. Then, for any sequence $\left\{q_{k}\right\}$ with $q_{k} \rightarrow 2_{+}$as $k \rightarrow \infty$, there exist $\left\{x_{k}\right\} \subset \mathbb{R}^{2}, \beta>0$ and a subsequence of $\left\{q_{k}\right\}$, still denoted by $\left\{q_{k}\right\}$, such that

$$
\frac{1}{\left\|\nabla u_{q_{k}}\right\|_{L^{2}\left(\mathbb{R}^{2}\right)}} u_{q_{k}}\left(\frac{x}{\left\|\nabla u_{q_{k}}\right\|_{L^{2}\left(\mathbb{R}^{2}\right)}}+x_{k}\right) \rightarrow \frac{\beta}{\|Q\|_{L^{2}\left(\mathbb{R}^{2}\right)}} Q(\beta x)
$$

strongly in $L^{2}\left(\mathbb{R}^{2}\right)$.

The proof of Theorem 1.2 is delicate. We commence with the estimate $E_{a, q}\left(u_{q}\right)$ for the mountain pass solution $u_{q}$. It is shown in section 4 that

$$
\frac{q-2}{2 q} \tau_{q}^{2} \leq E_{a, q}\left(u_{q}\right) \leq \frac{q-2}{2 q} \tau_{q}^{2}+\tau_{q}^{-2}\left(\frac{1}{2} \int_{\mathbb{R}^{2}}|x|^{2} \frac{|Q(x)|^{2}}{\|Q\|_{2}^{2}} d x+o(1)\right) \quad \text { as } \quad q \rightarrow 2_{+} .
$$

In contrast with subcritical or critical cases, such an estimate can not established for the supercritical case by simply choosing a suitable trail function. Instead, we need to construct a suitable path, and estimate energy of $E_{a, q}(u)$ on it. Then the proof of Theorem 1.2 is 
completed by the blow up analysis. This is different and more difficult than subcritical and critical cases because there is essentially no compactness for the sequence $\left\{u_{q}\right\}$.

Finally, we study the asymptotic behavior of $x_{k}$.

Theorem 1.3. Let $\left\{x_{k}\right\}$ be in Theorem 1.2. Then either $\liminf _{k \rightarrow \infty}\left|x_{k}\right| \rightarrow+\infty$; or there exists a subsequence of $\left\{x_{k}\right\}$, still denoted by $\left\{x_{k}\right\}$ such that

$$
x_{k} \rightarrow x_{0} \in \mathcal{Z}:=\left\{\left(b_{1} A, 0\right),\left(-b_{1} A, 0\right)\right\}
$$

with

$$
\tau_{q_{k}}\left(\left|x_{k}\right|_{b}-A\right) \rightarrow 0
$$

Moreover, in this case equation (1.11) holds with $\beta=1$, and $\tau_{q_{k}}^{-2}\left\|\nabla u_{q_{k}}\right\|_{L^{2}\left(\mathbb{R}^{2}\right)}^{2} \rightarrow 1$ as $k \rightarrow \infty$.

This paper is organized as follows. In section 2, we collect and prove some relevant results for future reference. Then, in section 3, we establish the existence of critical points of $E_{a, q}$. Finally, we analyze the limiting behavior of these critical points in section 4 .

\section{Preliminaries}

In this section, we collect and prove some relevant results for future reference. The following result is used frequently in literatures, we state it explicitly.

Lemma 2.1. Let $\varphi_{q} \geq 0$ be the unique radially symmetric positive solution of (1.8) with $2<q \leq 3$. Then, $\varphi_{q} \rightarrow Q$ strongly in $H^{1}\left(\mathbb{R}^{2}\right)$ as $q \rightarrow 2_{+}$, and there exist positive constants $C$ and $\delta$ independent of $q$ such that

$$
\varphi_{q}(x) \leq C e^{-\delta|x|} \text { for } x \in \mathbb{R}^{2} .
$$

In particular, $a_{q}^{*}=\|\varphi\|_{2}^{q} \rightarrow a^{*}=\|Q\|_{2}^{2}$ as $q \rightarrow 2_{+}$.

Let $\tilde{S}(1)=\left\{u \in H^{1}\left(\mathbb{R}^{2}\right):\|u\|_{2}^{2}=1\right\}$. Denote by $\left.E_{a, q}\right|_{V=0}(u)$ the energy functional without the trapping potential

$$
\left.E_{a, q}\right|_{V=0}(u)=\frac{1}{2} \int_{\mathbb{R}^{2}}|\nabla u|^{2} d x-\frac{a}{q+2} \int_{\mathbb{R}^{2}}|u|^{q+2} d x, \quad u \in H^{1}\left(\mathbb{R}^{2}\right) .
$$

We define

$$
\tilde{c}_{q}=\left.\inf _{g \in \Gamma} \max _{u \in g} E_{a, q}\right|_{V=0}(u),
$$

where

$$
\tilde{\Gamma}=\left\{g \in C([0,1], \tilde{S}(1)) \mid g(0)=u_{1}, g(1)=u_{2}\right\}
$$

for $u_{1}, u_{2} \in \tilde{S}(1)$ such that $\tilde{c}_{q}>\max \left\{\left.E_{a, q}\right|_{V=0}(g(0)),\left.E_{a, q}\right|_{V=0}(g(1))\right\}$. We know from Theorem 2.1 in [14] that for every $a>0$, there exists a unique positive critical point $\tilde{\varphi}_{q}$ of $\left.E_{a, q}\right|_{V=0}(u)$ constrained on $S(1)$ at the energy level $\tilde{c}_{q}$. Now, we give an explicit description of $\tilde{c}_{q}$ and $\tilde{\varphi}_{q}$ in terms of $a, a_{q}^{*}$ and $\varphi_{q}$. 
Lemma 2.2. Let $q>2$. Then

and

$$
\tilde{c}_{q}=\frac{q-2}{2 q} \tau_{q}^{2}
$$

$$
\tilde{\varphi}_{q}(x)=\frac{\tau_{q}}{\left\|\varphi_{q}\right\|_{2}} \varphi_{q}\left(\tau_{q} x\right) .
$$

Proof. It is know from [14] that

$$
\tilde{c}_{q}=\left.E_{a, q}\right|_{V=0}\left(\tilde{\varphi}_{q}\right), \quad\left\|\tilde{\varphi}_{q}\right\|_{2}^{2}=1
$$

and $\tilde{\varphi}_{q}$ satisfies

$$
-\Delta \tilde{\varphi}_{q}+\mu_{q} \tilde{\varphi}_{q}=a \tilde{\varphi}_{q}^{q+1},
$$

where $\mu_{q}$ is the Lagrange multiplier. Let $w_{q}=t \tilde{\varphi}_{q}(s x)$, where $s$ and $t$ satisfies

$$
s^{2} \mu_{q}=a t^{-q} s^{2}=\frac{2}{q} .
$$

Then, $w_{q}$ is a positive solution of (1.8). By the uniqueness of positive solutions of (1.8), we have $w_{q}=\varphi_{q}$. Since $\left\|\tilde{\varphi}_{q}\right\|_{2}^{2}=1$,

$$
1=\int_{\mathbb{R}^{2}}\left|\tilde{\varphi}_{q}\right|^{2} d x=t^{-2} s^{2} \int_{\mathbb{R}^{2}}\left|w_{q}\right|^{2} d x=t^{-2} s^{2}\left(a_{q}^{*}\right)^{\frac{2}{q}} .
$$

Solving

$$
a t^{-q} s^{2}=\frac{2}{q} \quad \text { and } \quad t^{-2} s^{2}\left(a_{q}^{*}\right)^{\frac{2}{q}}=1
$$

we obtain

Hence, we get

$$
t=\left(\frac{2 a_{q}^{*}}{q a}\right)^{\frac{1}{2-q}}\left(a_{q}^{*}\right)^{\frac{1}{q}}, \quad s=\left(\frac{2 a_{q}^{*}}{q a}\right)^{\frac{1}{2-q}}=\tau_{q}^{-1}
$$

$$
\tilde{\varphi}_{q}(x)=\frac{\tau_{q}}{\left\|\varphi_{q}\right\|_{2}} \varphi_{q}\left(\tau_{q} x\right)
$$

Substituting $\tilde{\varphi}_{q}$ into $\left.E_{a, q}\right|_{V=0}(u)$, we obtain

$$
\tilde{c}_{q}=\frac{q-2}{2 q}\left(\frac{2 a_{q}^{*}}{q a}\right)^{\frac{2}{q-2}} .
$$

Remark 2.1. It is known from Lemma 2.10 in [14] that $\tilde{c}_{q}$ can also be described by

$$
\tilde{c}_{q}=\left.\inf _{u \in \mathcal{Q}} E_{a, q}\right|_{V=0}(u),
$$

where

and

$$
\mathcal{Q}=\left\{u \in H^{1}\left(\mathbb{R}^{2}\right) \mid\|u\|_{2}^{2}=1, \tilde{Q}_{q}(u)=0\right\}
$$

$$
\tilde{Q}_{q}(u)=\int_{\mathbb{R}^{2}}|\nabla u|^{2} d x-\frac{q a}{q+2} \int_{\mathbb{R}^{2}}|u|^{q+2} d x
$$




\section{EXISTEnCE}

In this section, we show the existence of critical points of the functional $E_{a, q}(u)$ on the sphere

$$
S(1)=\left\{u \in \mathcal{H},\|u\|_{2}^{2}=1\right\},
$$

that is, we will prove Theorem 1.1. This will be done by a variant mountain pass theorem. Before we proceed further, we recall the following compactness lemma, which can be proved as that in [23].

Lemma 3.1. Suppose $V \in L_{l o c}\left(\mathbb{R}^{2}\right)$ and $\lim _{x \rightarrow \infty} V(x)=\infty$. Then the embedding $\mathcal{H} \hookrightarrow$ $L^{q}\left(\mathbb{R}^{2}\right)$ is compact for $q \in[2, \infty)$.

Proof of Theorem 1.1. Obviously, $E_{a, q}(u) \geq\left. E_{a, q}\right|_{V=0}(u)$ for every $u \in \mathcal{H}$. We claim that

$$
\left.\inf _{u \in R_{q}} E_{a, q}\right|_{V=0}(u) \geq \frac{q-2}{2 q} \tau_{q}^{2}
$$

where

which implies

$$
R_{q}=\left\{u \in S(1):\|\nabla u\|_{2}^{2}=\tau_{q}^{2}\right\}
$$

$$
\inf _{u \in R_{q}} E_{a, q}(u) \geq\left.\inf _{u \in R_{q}} E_{a, q}\right|_{V=0}(u) \geq \frac{q-2}{2 q} \tau_{q}^{2} .
$$

Indeed, by the Gagliardo-Nirenberg inequality (see [21]), we get

$$
\left.E_{a, q}\right|_{V=0}(u) \geq \frac{1}{2} \int_{\mathbb{R}^{2}}|\nabla u(x)|^{2} d x-\frac{a}{2 a_{q}^{*}}\left(\int_{\mathbb{R}^{2}}|\nabla u(x)|^{2} d x\right)^{\frac{q}{2}} \int_{\mathbb{R}^{2}}|u(x)|^{2} d x .
$$

Hence

$$
\left.\inf _{u \in R_{q}} E_{a, q}\right|_{V=0}(u) \geq \frac{1}{2} \tau_{q}^{2}-\frac{a}{2 a_{q}^{*}} \tau_{q}^{q}=\frac{q-2}{2 q} \tau_{q}^{2} .
$$

Let $\varphi \in C_{c}\left(\mathbb{R}^{2}\right)$ be a nonnegative function such that $\|\varphi\|_{2}^{2}=1$, and denote $\varphi^{t}(x)=t \varphi(t x)$. Then,

$$
E_{a, q}\left(\varphi^{t}\right)=\frac{1}{2} t^{2} \int_{\mathbb{R}^{2}}|\nabla \varphi(x)|^{2} d x+\frac{1}{2} \int_{\mathbb{R}^{2}} V\left(t^{-1} x\right)|\varphi(x)|^{2} d x-\frac{a t^{q}}{q+2} \int_{\mathbb{R}^{2}}|\varphi(x)|^{q+2} d x .
$$

Let $t_{1}=2^{(q-2)^{-2}}$. Since

$$
\lim _{q \rightarrow 2^{+}} \int_{\mathbb{R}^{2}} V\left(t_{1}^{-1} x\right)|\varphi(x)|^{2} d x=V(0)
$$

and

we have

$$
\lim _{q \rightarrow 2^{+}} \frac{\frac{a t_{1}^{q}}{q+2} \int_{\mathbb{R}^{2}}|\varphi(x)|^{q+2} d x}{\frac{1}{2} t_{1}^{2} \int_{\mathbb{R}^{2}}|\nabla \varphi(x)|^{2} d x}=\lim _{q \rightarrow 2^{+}} \frac{2 a}{q+2} \frac{\|\varphi\|_{q+2}^{q+2} t_{1}^{q-2}}{\|\nabla \varphi\|_{2}^{2}}=+\infty
$$

$$
E\left(\varphi^{t_{1}}\right) \leq 2 V(0)-\frac{a t_{1}^{q}}{2(q+2)}\|\varphi\|_{q+2}^{q+2}=2 V(0)-\frac{a 2^{q(q-2)^{-2}}}{2(q+2)}\|\varphi\|_{q+2}^{q+2}
$$


Hence

$$
\lim _{q \rightarrow 2_{+}} E_{a, q}\left(\varphi^{t_{1}}\right)=-\infty
$$

It implies that there exists $\varepsilon_{0}>0$ such that

$$
E_{a, q}\left(\varphi^{t_{1}}\right)<0
$$

if $q \in\left(2,2+\varepsilon_{0}\right)$. Let

$$
c_{q}=\inf _{g \in \Gamma_{q}} \max _{t \in[0,1]} E_{a, q}(g(t)),
$$

where

$$
\Gamma_{q}=\left\{g \in C([0,1], S(1)): g(0)=\varphi, \quad g(1)=\varphi^{t_{1}}\right\} .
$$

Since $\lim _{q \rightarrow 2_{+}} \frac{q-2}{2 q} \tau_{q}^{2}=+\infty$, we deduce

$$
\lim _{q \rightarrow 2^{+}} \frac{\left\|\nabla \varphi^{t_{1}}\right\|_{2}^{2}}{\tau_{q}^{2}}=\lim _{q \rightarrow 2^{+}} \frac{t_{1}^{2}\|\nabla \varphi\|_{2}^{2}}{\tau_{q}^{2}}=\lim _{q \rightarrow 2^{+}}\left(\frac{2^{(q-2)^{-1}} q a}{2 a_{q}^{*}}\right)^{2(q-2)^{-1}}\|\nabla \varphi\|_{2}^{2}=+\infty,
$$

that is,

$$
\lim _{q \rightarrow 2^{+}} \frac{t_{1}^{2}}{\tau_{q}^{2}}=+\infty .
$$

Hence, by (3.2),(3.5) for any $g \in \Gamma_{q}$, if $q>2$ and $q$ is close to 2, there holds

$$
\max _{t \in[0,1]} E_{a, q}(g(t)) \geq\left.\inf _{u \in R_{q}} E_{a, q}\right|_{V=0}(u) \geq \frac{q-2}{2 q} \tau_{q}^{2}>\max \left\{E_{a, q}(\varphi), E_{a, q}\left(\varphi^{t_{1}}\right)\right\} .
$$

As a result,

$$
c_{q} \geq \frac{q-2}{2 q} \tau_{q}^{2}>\max \left\{E_{a, q}(\varphi), E_{a, q}\left(\varphi^{t_{1}}\right)\right\} .
$$

Equations (3.5) and (3.6) indicate that the functional $E_{a, q}$ has the mountain pass geometry, and then there exists a $(P S)$ sequence of $E_{a, q}$ at the mountain pass level. However, such a $(P S)$ sequence may fail to be bounded. In order to bound (PS) sequence, we use the following variant mountain pass theorem.

Let $H(u, s)=e^{s} u\left(e^{s} x\right)$. The functional $\mathcal{E}_{a, q}: \mathcal{H} \times \mathbb{R} \rightarrow \mathbb{R}$ is defined as

$$
\begin{aligned}
\mathcal{E}_{a, q}(u, s) & =: E_{a, q}(H(u, s)) \\
& =\frac{1}{2} e^{2 s} \int_{\mathbb{R}^{2}}|\nabla u(x)|^{2} d x+\frac{1}{2} \int_{\mathbb{R}^{2}} V\left(e^{-s} x\right)|u(x)|^{2} d x-\frac{a}{q+2} e^{s q} \int_{\mathbb{R}^{2}}|u(x)|^{q+2} d x .
\end{aligned}
$$

Denote the set of paths by

$$
\mathcal{P}_{q}=\left\{\gamma \in C([0,1], S(1) \times \mathbb{R}): \gamma(0)=(\varphi, 0), \gamma(1)=\left(\varphi^{t_{1}}, 0\right)\right\}
$$

and define

$$
b_{q}=\inf _{\gamma \in \mathcal{P}_{q}} \max _{t \in[0,1]} \mathcal{E}_{a, q}(\gamma(t))=\inf _{\gamma \in \mathcal{P}_{q}} \max _{t \in[0,1]} E_{a, q}(H(\gamma(t))) .
$$

Since $\Gamma_{q}=\left\{H(\gamma): \gamma \in \mathcal{P}_{q}\right\}$, we get

$$
b_{q}=c_{q} .
$$

By $(3.6), \mathcal{E}_{a, q}(\gamma(0))=E_{a, q}(\varphi)$ and $\mathcal{E}_{a, q}(\gamma(1))=E_{a, q}\left(\varphi^{t_{1}}\right)$, we have

$$
b_{q}=c_{q}>\max \left\{E_{a, q}(\varphi), E_{a, q}\left(\varphi^{t_{1}}\right)\right\}=\max \left\{\mathcal{E}_{a, q}(\gamma(0)), \mathcal{E}_{a, q}(\gamma(1))\right\} .
$$


Let $Y=\mathcal{H} \times \mathbb{R}$ be the space with the norm

$$
\|(u, s)\|_{Y}^{2}=\|(u, s)\|_{\mathcal{H}}^{2}+\|s\|_{\mathbb{R}}^{2} \text { for any }(u, s) \in Y .
$$

Then, $S(1) \times \mathbb{R}$ is a submanifold of $Y$ of codimension 1 and its tangent subspace at a given point $(u, s) \in S(1) \times \mathbb{R}$ is

$$
T_{(u, s)}(S(1) \times \mathbb{R})=\left\{\left(v_{1}, s_{1}\right) \in Y:\left\langle v_{1}, u\right\rangle_{L^{2}\left(\mathbb{R}^{2}\right)}=0\right\} .
$$

Denote by $\left.\mathcal{E}_{a, q}\right|_{S(1) \times \mathbb{R}}$ the trace of $\mathcal{E}_{a, q}$ on $S(1) \times \mathbb{R}$. Then, $\left.\mathcal{E}_{a, q}\right|_{S(1) \times \mathbb{R}}$ is a $C^{1}$ functional on $S(1) \times \mathbb{R}$, and for any $(u, s) \in S(1) \times \mathbb{R}$,

$$
\left\langle\left.\mathcal{E}_{a, q}^{\prime}\right|_{S(1) \times \mathbb{R}}((u, s)), w\right\rangle=\left\langle\mathcal{E}_{a, q}^{\prime}((u, s)), w\right\rangle \text { for } w \in T_{(u, s)}(S(1) \times \mathbb{R}) .
$$

Moreover,

$$
\left.\mathcal{E}_{a, q}^{\prime}\right|_{S(1) \times \mathbb{R}} \in\left[T_{(u, s)}(S(1) \times \mathbb{R})\right]^{-1} .
$$

By (3.8), there exists $\left\{\left(g_{n}, 0\right)\right\} \subset \mathcal{P}_{q}$ such that

$$
c_{q}=\lim _{n \rightarrow \infty} \sup _{g_{n}} E_{a, q}\left(g_{n}\right)=\lim _{n \rightarrow \infty} \sup _{\left(g_{n}, 0\right)} \mathcal{E}_{a, q}\left(g_{n}, 0\right)=b_{q} .
$$

Because $E_{a, q}\left(\left|g_{n}\right|\right) \leq E_{a, q}\left(g_{n}\right)$, let $\gamma_{n}=\left(\left|g_{n}\right|, 0\right)$, we have

$$
\lim _{n \rightarrow \infty} \sup _{\gamma_{n}} \mathcal{E}_{a, q}\left(\gamma_{n}\right)=b_{q}=c_{q} .
$$

By Theorem 3.2 in [11], there exists $\left\{\left(w_{n}, s_{n}\right)\right\} \subset S(1) \times \mathbb{R}$ such that

$$
\begin{gathered}
\lim _{n \rightarrow \infty} \mathcal{E}_{a, q}\left(w_{n}, s_{n}\right)=b_{q}=c_{q}, \\
\lim _{n \rightarrow \infty}\left\|\left.\mathcal{E}_{a, q}^{\prime}\right|_{S(1) \times \mathbb{R}}\left(w_{n}, s_{n}\right)\right\|_{\mathcal{Z}_{n}}=0 \quad \text { with } \quad \mathcal{Z}_{n}=\left[T_{\left(w_{n}, s_{n}\right)}(S(1) \times \mathbb{R})\right]^{-1},
\end{gathered}
$$

and

$$
\lim _{n \rightarrow \infty} \operatorname{dist}\left(\left(w_{n}, s_{n}\right),\left(\left|g_{n}\right|, 0\right)\right)=0 .
$$

Let $u_{n}=H\left(w_{n}, s_{n}\right)$. Choose $v_{n} \in\left|g_{n}\right|$ such that

$$
\left\|u_{n}-v_{n}\right\|_{\mathcal{H}}^{2}+\left|s_{n}\right|^{2}=\left(\operatorname{dist}\left(\left(w_{n}, s_{n}\right),\left(\left|g_{n}\right|, 0\right)\right)\right)^{2} .
$$

Then

$$
\lim _{n \rightarrow \infty}\left\|u_{n}-v_{n}\right\|_{\mathcal{H}}^{2}+\left|s_{n}\right|^{2}=0
$$

By (3.11),

$$
\lim _{n \rightarrow \infty} E_{a, q}\left(u_{n}\right)=\lim _{n \rightarrow \infty} \mathcal{E}_{a, q}\left(w_{n}, s_{n}\right)=c_{q} .
$$

For any $(\varphi, \tau) \in T_{(u, s)}(S(1) \times \mathbb{R})$ with $(u, s) \in S(1) \times \mathbb{R}$, we claim that

$$
\left\langle\left.\mathcal{E}_{a, q}^{\prime}\right|_{S(1) \times \mathbb{R}}(u, s),(\varphi, \tau)\right\rangle=\left\langle\left. E_{a, q}^{\prime}\right|_{S(1)}(H(u, s)), H(\varphi, s)\right\rangle+Q_{q}(H(u, s)) \tau,
$$

where

$$
Q_{q}(u)=\int_{\mathbb{R}^{2}}|\nabla u(x)|^{2} d x-\frac{1}{2} \int_{\mathbb{R}^{2}} x \cdot \nabla V(x)|u(x)|^{2} d x-\frac{q a}{q+2} \int_{\mathbb{R}^{2}}|u(x)|^{q+2} d x .
$$


Indeed,

$$
\begin{aligned}
& \left\langle\left.\mathcal{E}_{a, q}^{\prime}\right|_{S(1) \times \mathbb{R}}(u, s),(\varphi, \tau)\right\rangle \\
& =\left\langle\mathcal{E}_{a, q}^{\prime}(u, s),(\varphi, \tau)\right\rangle \\
& =\lim _{t \rightarrow 0} t^{-1}\left[\mathcal{E}_{a, q}(u+t \varphi, s+t \tau)-\mathcal{E}_{a, q}(u, s)\right] \\
& =\lim _{t \rightarrow 0} t^{-1}\left[\mathcal{E}_{a, q}(u+t \varphi, s+t \tau)-\mathcal{E}_{a, q}(u, s+t \tau)+\mathcal{E}_{a, q}(u, s)-\mathcal{E}_{a, q}(u+t \varphi, s)\right] \\
& +\lim _{t \rightarrow 0} t^{-1}\left[\mathcal{E}_{a, q}(u, s+t \tau)-\mathcal{E}_{a, q}(u, s)\right]+\lim _{t \rightarrow 0} t^{-1}\left[\mathcal{E}_{a, q}(u+t \varphi, s)-\mathcal{E}_{a, q}(u, s)\right] \\
& :=I_{1}+I_{2}+I_{3} .
\end{aligned}
$$

We deduce that

$$
\begin{aligned}
I_{1}= & \lim _{t \rightarrow 0} t^{-1}\left\{\frac{1}{2}\left(e^{2(s+t \tau)}-e^{2 s}\right) \int_{\mathbb{R}^{2}}\left(|\nabla u+t \nabla \varphi|^{2}-|\nabla u|^{2}\right) d x\right. \\
& +\frac{1}{2} \int_{\mathbb{R}^{2}}\left[\left(\frac{|x|_{b}}{s+t \tau}-A\right)^{2}-\left(\frac{|x|_{b}}{s}-A\right)^{2}\right]\left[|u+t \varphi|^{2}-|u|^{2}\right] d x \\
& \left.-\frac{a}{q+2}\left[e^{q(s+t \tau)}-e^{q s}\right] \int_{\mathbb{R}^{2}}\left[|u+t \varphi|^{q+2}-|u|^{q+2}\right] d x\right\} \\
= & \lim _{t \rightarrow 0} t^{-1}\left\{\frac{1}{2} e^{2 s}\left(e^{2 t \tau}-1\right) \int_{\mathbb{R}^{2}}\left(2 t \nabla u \cdot \nabla \varphi+t^{2}|\nabla \varphi|^{2}\right) d x\right. \\
& -\frac{t \tau}{2 s(s+t \tau)} \int_{\mathbb{R}^{2}}|x|_{b}\left(\frac{|x|_{b}}{s+t \tau}+\frac{|x|_{b}}{s}-2 A\right)\left(2 t u \varphi+t^{2} \varphi^{2}\right) d x \\
& \left.-\frac{a}{q+2} e^{q s}\left(e^{q t \tau}-1\right) \int_{\mathbb{R}^{2}}\left[|u+t \varphi|^{q+2}-|u|^{q+2}\right] d x\right\} \\
= & 0,
\end{aligned}
$$

and

$$
I_{2}=\lim _{t \rightarrow 0} t^{-1}\left[E_{a, q}\left(e^{s+t \tau} u\left(e^{s+t \tau} x\right)\right)-E_{a, q}\left(e^{s} u\left(e^{s} x\right)\right)\right]=\tau Q_{q}\left(e^{s} u\left(e^{s} x\right)\right) .
$$

Since $\langle\varphi, u\rangle_{L^{2}\left(\mathbb{R}^{2}\right)}=0$, we have

$$
e^{s} \varphi\left(e^{s} x\right) \in T_{e^{s} u\left(e^{s} x\right)} S(1):=\left\{\psi \in \mathcal{H}:\left\langle\psi, e^{s} u\left(e^{s} x\right)\right\rangle_{L^{2}\left(\mathbb{R}^{2}\right)}=0\right\}
$$

and

$$
\begin{aligned}
I_{3} & =\lim _{t \rightarrow 0} t^{-1}\left[E_{a, q}\left(e^{s} u\left(e^{s} x\right)+t e^{s} \varphi\left(e^{s} x\right)\right)-E_{a, q}\left(e^{s} u\left(e^{s} x\right)\right)\right] \\
& =\left\langle E_{a, q}^{\prime}\left(e^{s} u\left(e^{s} x\right)\right), e^{s} \varphi\left(e^{s} x\right)\right\rangle \\
& =\left\langle\left. E_{a, q}^{\prime}\right|_{S(1)}\left(e^{s} u\left(e^{s} x\right)\right), e^{s} \varphi\left(e^{s} x\right)\right\rangle .
\end{aligned}
$$

As a result, the claim (3.15) holds.

For any $(\varphi, \tau) \in T_{\left(w_{n}, \tau_{n}\right)}(S(1) \times \mathbb{R})$, we infer from (3.12) and (3.15) that,

$$
\left\langle\left. E_{a, q}^{\prime}\right|_{S(1)}\left(u_{n}\right), H\left(\varphi, s_{n}\right)\right\rangle+Q_{q}\left(u_{n}\right) \tau=o\left(\|(\varphi, \tau)\|_{Y}^{2}\right) .
$$

Choosing particularly $(\varphi, \tau)=(0,1)$ in $(3.17)$, we obtain

$$
Q_{q}\left(u_{n}\right) \rightarrow 0 \text { as } n \rightarrow \infty \text {. }
$$


On the other hand, for any $\psi \in T_{u_{n}} S(1)$, we may choose $\varphi$ such that $\psi(x)=e^{s_{n}} \varphi\left(e^{s_{n}} x\right)$. Therefore,

$$
\left(\varphi, s_{n}\right) \in T_{\left(w_{n}, s_{n}\right)}(S(1) \times \mathbb{R}) .
$$

Taking $\tau=0$ in (3.17), we have

$$
\left\langle\left. E_{a, q}^{\prime}\right|_{S(1)}\left(u_{n}\right), \psi\right\rangle=o\left(\|\varphi\|_{\mathcal{H}}^{2}\right) .
$$

By (3.13),

$$
\left|s_{n}\right| \rightarrow 0
$$

as $n \rightarrow \infty$ and $\|\psi\|_{\mathcal{H}} \leq 2\|\varphi\|_{\mathcal{H}}$. Consequently,

$$
\left\langle\left. E_{a, q}^{\prime}\right|_{S(1)}\left(u_{n}\right), \psi\right\rangle=o\left(\|\psi\|_{\mathcal{H}}^{2}\right),
$$

that is

$$
\left\|\left.E_{a, q}^{\prime}\right|_{S(1)}\left(u_{n}\right)\right\|_{\mathcal{H}^{-1}} \rightarrow 0 \text { as } n \rightarrow \infty \text {. }
$$

The identity

$\frac{q-2}{2} \int_{\mathbb{R}^{2}}\left|\nabla u_{n}(x)\right|^{2} d x+\frac{1}{2} \int_{\mathbb{R}^{2}}\left[q\left(|x|_{b}-A\right)^{2}+2|x|_{b}\left(|x|_{b}-A\right)\right]\left|u_{n}(x)\right|^{2} d x=q E_{a, q}\left(u_{n}\right)-Q_{q}\left(u_{n}\right)$

and $\left\|u_{n}\right\|_{2}^{2}=1$ yield

$$
\begin{aligned}
& q E_{a, q}\left(u_{n}\right)-Q_{q}\left(u_{n}\right) \\
& \geq \frac{q-2}{2} \int_{\mathbb{R}^{2}}\left|\nabla u_{n}(x)\right|^{2} d x+\frac{1}{2} \int_{\mathbb{R}^{2}} q\left(|x|_{b}-A\right)^{2}\left|u_{n}(x)\right|^{2} d x+\frac{1}{2} \int_{\mathbb{R}^{2}}|x|_{b}^{2}\left|u_{n}(x)\right|^{2} d x-\frac{1}{2} A^{2} .
\end{aligned}
$$

This together with (3.14) and (3.18) implies that $\left\{u_{n}\right\}$ is bounded in $\mathcal{H}$. By Lemma 3.1, we may assume that

$$
u_{n} \rightarrow u_{q} \in \mathcal{H} \text { weakly in } \mathcal{H} \text {; }
$$

and

$$
u_{n} \rightarrow u_{q} \text { strongly in } L^{\gamma}\left(\mathbb{R}^{2}\right) \text { for any } \gamma \geq 2 .
$$

We know from (3.17) and Lemma 3 in [5] that there exists a $\mu_{q}^{n}$ such that

$$
-\Delta u_{n}+V u_{n}-a\left|u_{n}\right|^{q} u_{n}-\mu_{q}^{n} u_{n} \rightarrow 0 \text { in } \mathcal{H}^{-1},
$$

and then,

$$
\mu_{q}^{n}=\int_{\mathbb{R}^{2}}\left|\nabla u_{n}(x)\right|^{2} d x+\int_{\mathbb{R}^{2}} V(x)\left|u_{n}(x)\right|^{2} d x-a \int_{\mathbb{R}^{2}}\left|u_{n}(x)\right|^{q+2} d x+o\left(\left\|u_{n}\right\|_{\mathcal{H}}\right),
$$

which is bounded. Without loss of generality, we assume that $\mu_{q}^{n} \rightarrow \mu_{q}$ as $n \rightarrow \infty$. Passing the limit $n \rightarrow \infty$ in (3.24), we obtain

$$
-\Delta u_{q}+V u_{q}-a\left|u_{q}\right|^{q} u_{q}-\mu_{q} u_{q}=0 .
$$


By (3.25) and (3.26), we have

$$
\begin{aligned}
& \lim _{n \rightarrow \infty}\left(\int_{\mathbb{R}^{2}}\left|\nabla u_{n}(x)\right|^{2} d x+\int_{\mathbb{R}^{2}} V(x)\left|u_{n}(x)\right|^{2} d x\right) \\
& =a \int_{\mathbb{R}^{2}}\left|u_{q}\right|^{q+2} d x+\mu_{q} \\
& =\int_{\mathbb{R}^{2}}\left|\nabla u_{q}(x)\right|^{2}+\int_{\mathbb{R}^{2}} V(x)\left|u_{q}(x)\right|^{2} d x,
\end{aligned}
$$

namely,

$$
\lim _{n \rightarrow \infty}\left\|u_{n}\right\|_{\mathcal{H}}=\left\|u_{q}\right\|_{\mathcal{H}}
$$

By (3.13) and (3.19), we obtain $v_{n} \rightarrow u_{q}$ in $\mathcal{H}$. Note that $v_{n}$ is positive, we get $u_{q} \geq 0$ and $u_{q}$ is a solution of (3.26). Then the strong maximum principle implies $u_{q}>0$.

Since $u_{n} \rightarrow u_{q}$ in $\mathcal{H}$, by (3.14) and (3.18),

$$
E_{a, q}\left(u_{q}\right)=c_{q}
$$

and

$$
Q_{q}\left(u_{q}\right)=\int_{\mathbb{R}^{2}}\left|\nabla u_{q}(x)\right|^{2} d x-\int_{\mathbb{R}^{2}}|x|_{b}\left(|x|_{b}-A\right)\left|u_{q}(x)\right|^{2} d x-\frac{q a}{q+2} \int_{\mathbb{R}^{2}}\left|u_{q}(x)\right|^{q+2} d x=0 .
$$

The proof is complete.

\section{EnERGy ESTimates AND ASYMPtotic BeHAVior}

In this section, we first establish an asymptotic expansion of the energy $E_{a, q}\left(u_{q}\right)$ at the mountain pass point $u_{q}$. This relies on, among other things, a carefully choosing a path. Next, we study asymptotic behavior of $u_{q}$ as $q \rightarrow 2_{+}$and prove Theorem 1.2 and Theorem 1.3 .

Proposition 4.1. There holds

$$
\frac{q-2}{2 q} \tau_{q}^{2} \leq E_{a, q}\left(u_{q}\right) \leq \frac{q-2}{2 q} \tau_{q}^{2}+\tau_{q}^{-2}\left(\frac{1}{2} \int_{\mathbb{R}^{2}}|x|^{2} \frac{|Q(x)|^{2}}{\|Q\|_{2}^{2}} d x+o(1)\right) \quad \text { as } \quad q \rightarrow 2_{+} .
$$

Proof. By (3.6) and $c_{q}=E_{a, q}\left(u_{q}\right)$, it suffices to prove that

$$
E_{a, q}\left(u_{q}\right) \leq \frac{q-2}{2 q} \tau_{q}^{2}+\tau_{q}^{-2}\left(\frac{1}{2} \int_{\mathbb{R}^{2}}|x|^{2} \frac{|Q(x)|^{2}}{\|Q\|_{2}^{2}} d x+o(1)\right) \text { as } q \rightarrow 2_{+} .
$$

To this purpose, we will construct a path $g \in \Gamma_{q}$ linking $\varphi$ and $\varphi^{t_{1}}$ so that

$$
E_{a, q}\left(u_{q}\right) \leq \max _{t \in[0,1]} E_{a, q}(g(t)) \leq \frac{q-2}{2 q} \tau_{q}^{2}+\tau_{q}^{-2}\left(\frac{1}{2} \int_{\mathbb{R}^{2}}|x|^{2} \frac{|Q(x)|^{2}}{\|Q\|_{2}^{2}} d x+o(1)\right) .
$$

The path $g$ is constructed in three parts. 
First, we construct a path $g_{1}$ linking $\varphi$ to some $w_{q}^{\tilde{t}_{0}}$ and estimate $E_{a, q}\left(g_{1}(s)\right)$. Let $\varphi_{q}$ be the positive solution of (1.8). Denote

$$
w_{q}(x)=\frac{\tau_{q}}{\left\|\varphi_{q}\right\|_{2}} \varphi_{q}\left(\tau_{q}\left(x-x_{0}\right)\right.
$$

with $x_{0} \in \mathcal{Z}$. By Lemma 8.12 in [6], $\varphi_{q}$ satisfies

$$
\int_{\mathbb{R}^{2}}\left|\nabla \varphi_{q}(x)\right|^{2} d x=\int_{\mathbb{R}^{2}}\left|\varphi_{q}(x)\right|^{2} d x=\frac{2}{q+2} \int_{\mathbb{R}^{2}}\left|\varphi_{q}(x)\right|^{q+2} d x .
$$

Note that $a_{q}^{*}=\left\|\varphi_{q}\right\|_{2}^{q}$, we obtain

$$
\int_{\mathbb{R}^{2}}\left|\nabla w_{q}(x)\right|^{2} d x=\tau_{q}^{2}
$$

and

$$
\int_{\mathbb{R}^{2}}\left|w_{q}(x)\right|^{q+2} d x=\frac{q+2}{2 a_{q}^{*}} \tau_{q}^{q}
$$

Choosing $\tilde{t}_{0}=\sqrt{\frac{q-2}{12 q}}$ such that $w_{q}^{\tilde{t}_{0}}(x)=\tilde{t}_{0} w_{q}\left(\tilde{t}_{0} x\right)$ satisfies

$$
\int_{\mathbb{R}^{2}}\left|\nabla w_{q}^{\tilde{t}_{0}}\right|^{2} d x=\frac{q-2}{12 q} \tau_{q}^{2}
$$

we define

$$
g_{1}(s):=\frac{s w_{q}^{\tilde{t}_{0}}+(1-s) \varphi}{\left\|s w_{q}^{\tilde{t}_{0}}+(1-s) \varphi\right\|_{2}}, \quad s \in[0,1],
$$

where $\varphi \in C_{c}\left(\mathbb{R}^{2}\right)$ is a nonnegative function with $\|\varphi\|_{2}^{2}=1$ given in $\Gamma_{q}$.

By (4.4) and the fact that $\left\|w_{q}^{\tilde{t}_{0}}\right\|_{2}^{2}=\|\varphi\|_{2}^{2}=1$, we have

$$
\begin{aligned}
\frac{1}{2}\left\|\nabla g_{1}(s)\right\|_{2}^{2} & \leq 2\left(s^{2}\left\|\nabla w_{q}^{\tilde{t}_{0}}\right\|_{2}^{2}+(1-s)^{2}\|\nabla \varphi\|_{2}^{2}\right) \\
& \leq 2\left(\left\|\nabla w_{q}^{\tilde{t}_{0}}\right\|_{2}^{2}+\|\nabla \varphi\|_{2}^{2}\right) \\
& \leq \frac{(q-2)}{4 q} \tau_{q}^{2}
\end{aligned}
$$

since $\lim _{q \rightarrow 2^{+}} \frac{q-2}{q} \tau_{q}^{2}=+\infty$. On the other hand,

$$
\int_{\mathbb{R}^{2}} V(x)\left|g_{1}(x)\right|^{2} d x \leq 4\left(\int_{\mathbb{R}^{2}} V(x)\left|w_{q}^{\tilde{t}_{0}}\right|^{2} d x+\int_{\mathbb{R}^{2}} V(x)|\varphi|^{2} d x\right) .
$$

By Lemma 2.1,

$$
\varphi_{q}(x) \leq C e^{-\delta|x|}
$$


where $C>0, \delta>0$ independent of $q$, and Lebesgue dominated theorem,

$$
\begin{aligned}
\int_{\mathbb{R}^{2}} V(x)\left|w_{q}^{\tilde{t}_{0}}(x)\right|^{2} d x & =\int_{\mathbb{R}^{2}}\left(\tilde{t}_{0}^{-1}\left|\tau_{q}^{-1} y+x_{0}\right|_{b}-A\right)^{2} \frac{\left|\varphi_{q}(y)\right|^{2}}{\left\|\varphi_{q}\right\|_{2}^{2}} d y \\
& \leq 2\left(\tilde{t}_{0}^{-2}\left\|\varphi_{q}\right\|_{2}^{-2} \int_{\mathbb{R}^{2}}\left|\tau_{q}^{-1} y+x_{0}\right|_{b}^{2}\left|\varphi_{q}(y)\right|^{2} d y+A^{2}\right) \\
& =2\left(\tilde{t}_{0}^{-2}\left(\|Q\|_{2}^{-2} \int_{\mathbb{R}^{2}}\left|x_{0}\right|_{b}^{2}|Q(y)|^{2} d y+o(1)\right)+A^{2}\right] \\
& =2\left[\tilde{t}_{0}^{-2}\left(A^{2}+o(1)\right)+A^{2}\right] \\
& \leq 4 A^{2} \tilde{t}_{0}^{-2} \text { as } q \rightarrow 2_{+} .
\end{aligned}
$$

Since $\tilde{t}_{0}^{2}=\frac{q-2}{12 q},(4.5)$ and $(4.7)$ yield

$$
\begin{aligned}
E_{a, q} & \leq \frac{3(q-2)}{12 q} \tau_{q}^{2}+\frac{48 q A^{2}}{q-2} \\
& \leq \frac{(q-2)}{3 q} \tau_{q}^{2}
\end{aligned}
$$

for $q>2$ and $q$ close to 2 .

Next, we construct the second part $g_{2}$ of the path $g$. Choose $\tilde{t}_{1}=\tau_{q}^{-1} t_{1}:=\tau_{q}^{-1} 2^{(q-2)^{-2}}$. Let

$$
g_{2}(s)=\frac{s w_{q}^{\tilde{t}_{1}}+(1-s) \varphi^{t_{1}}}{\left\|s w_{q}^{\tilde{t}_{1}}+(1-s) \varphi^{t_{1}}\right\|_{2}}, \quad s \in[0,1] .
$$

By (4.2), (4.3) and $\left\|w_{q}^{\tilde{t}_{1}}\right\|_{2}^{2}=\left\|\varphi^{t_{1}}\right\|_{2}^{2}=1$, we have

$$
\begin{aligned}
\frac{1}{2}\left\|\nabla g_{2}(s)\right\|_{2}^{2} & \leq 2 s^{2}\left\|\nabla w_{q}^{\tilde{t}_{1}}\right\|_{2}^{2}+2(1-s)^{2}\left\|\nabla \varphi^{t_{1}}\right\|_{2}^{2} \\
& =2^{\frac{2}{(q-2)^{2}}+1} s^{2}+\|\nabla \varphi\|_{2}^{2} 2^{\frac{2}{(q-2)^{2}}+1}(1-s)^{2}
\end{aligned}
$$

and

$$
\begin{aligned}
\frac{a}{q+2} \int_{\mathbb{R}^{2}}\left|g_{2}(s)\right|^{q+2} d x & \geq \frac{4^{-q-2} a}{q+2} \int_{\mathbb{R}^{2}}\left|s w_{q}^{\tilde{t}_{1}}+(1-s) \varphi^{t_{1}}\right|^{q+2} d x \\
& \geq \frac{4^{-q-3} a}{q+2}\left[\int_{\mathbb{R}^{2}}\left|s w_{q}^{\tilde{t}_{1}}\right|^{q+2} d x+\int_{\mathbb{R}^{2}}\left|(1-s) \varphi^{t_{1}}\right|^{q+2} d x\right] \\
& =\frac{4^{-q-3} a}{q+2}\left[\frac{q+2}{2 a_{q}^{*}} s^{q+2} 2^{\frac{q}{(q-2)^{2}}}+(1-s)^{q+2} 2^{\frac{q}{(q-2)^{2}}} \int_{\mathbb{R}^{2}}|\varphi|^{q+2} d x\right] .
\end{aligned}
$$

If $0 \leq s \leq \frac{1}{2}$, then

$$
\begin{aligned}
\frac{1}{2}\left\|\nabla g_{2}(s)\right\|_{2}^{2}-\frac{a}{q+2} \int_{\mathbb{R}^{2}}\left|g_{2}(s)\right|^{q+2} d x & \leq C_{1} 2^{\frac{2}{(q-2)^{2}}}-C_{2} \frac{4^{-q-3} a}{q+2} 2^{\frac{q}{(q-2)^{2}}} \\
& \leq-C_{2} \frac{4^{-q-3} a}{2(q+2)} 2^{\frac{q}{(q-2)^{2}}} .
\end{aligned}
$$


Similarly, the inequality (4.11) holds for $\frac{1}{2} \leq s \leq 1$. We may show as (4.7) that

$$
\int_{\mathbb{R}^{2}} V(x)\left|g_{2}(s)\right|^{2} d x \leq C .
$$

Therefore, we deduce from from (4.11) and (4.12) that

$$
E_{a, q}\left(g_{2}(s)\right)<0
$$

for $q>2$ and close to 2 .

Finally, we construct a path linking $w_{q}^{\tilde{t}_{0}}$ and $w_{q}^{\tilde{t}_{1}}$. Since

$$
\left\|\nabla w_{q}^{\tilde{t}_{0}}\right\|_{2}^{2}=\frac{q-2}{12 q} \tau_{q}^{2} \text { and }\left\|\nabla w_{q}^{\tilde{t}_{1}}\right\|_{2}^{2}=2^{\frac{2}{(q-2)^{2}}},
$$

there exists a $\tilde{t}_{2} \in\left(\tilde{t}_{0}, \tilde{t}_{1}\right)$ such that $\left\|\nabla w_{q}^{\tilde{t}_{2}}\right\|_{2}^{2}=\tau_{q}^{2}$. By $(3.2)$, we have

$$
E_{a, q}\left(w_{q}^{\tilde{t}_{2}}\right) \geq \frac{q-2}{2 q} \tau_{q}^{2}
$$

Let

$$
g_{3}(s)=w_{q}^{s \tilde{t}_{0}+(1-s) \tilde{t}_{1}}, \quad s \in[0,1] .
$$

Now, we define a path $g$ by $g_{1}, g_{2}$ and $g_{3}$, that is,

$$
g(s)= \begin{cases}g_{1}(3 s), & \text { if } 0 \leq s \leq \frac{1}{3} \\ g_{3}(3 s-1), & \text { if } \frac{1}{3} \leq s \leq \frac{2}{3} \\ g_{2}(3 s-2), & \text { if } \frac{2}{3} \leq s \leq 1\end{cases}
$$

Obviously, $g \in \Gamma_{q}$.

It follows from (4.8), (4.13) and (4.14) that

$$
\begin{aligned}
\max _{0 \leq s \leq 1} E_{a, q}(g(s)) & =\max _{\frac{1}{3} \leq s \leq \frac{2}{3}} E_{a, q}\left(g_{3}(3 s-1)\right) \\
& =\max _{\tilde{t}_{0} \leq t \leq \tilde{t}_{1}} E_{a, q}\left(w_{q}^{t}\right) .
\end{aligned}
$$

Let $f(t)=E_{a, q}\left(w_{q}^{t}\right)$. It implies by (4.2) and (4.3) that

$$
f(t)=\frac{1}{2} \tau_{q}^{2} t^{2}-\frac{1}{q} \tau_{q}^{2} t^{q}+\frac{1}{2} \int_{\mathbb{R}^{2}} V\left(t^{-1} x\right)\left|w_{q}(x)\right|^{2} d x .
$$

By (4.8), (4.13) and (4.14), there exists $t_{q} \in\left(\tilde{t}_{0}, \tilde{t}_{1}\right)$ such that

$$
f\left(t_{q}\right)=\max _{t \in\left(\tilde{t}_{0}, \tilde{t}_{1}\right)} f(t)
$$

and

$$
f^{\prime}\left(t_{q}\right)=\tau_{q}^{2} t_{q}-\tau_{q}^{2} t_{q}^{q-1}-\frac{1}{t_{q}^{3}} \int_{\mathbb{R}^{2}}|x|_{b}^{2}\left|w_{q}(x)\right|^{2} d x+\frac{A}{t_{q}^{2}} \int_{\mathbb{R}^{2}}|x|_{b}\left|w_{q}(x)\right|^{2} d x
$$


By Lemma 2.1 and the Lebegue dominated theorem, we have

$$
\begin{aligned}
\int_{\mathbb{R}^{2}}|x|_{b}^{2}\left|w_{q}(x)\right|^{2} d x & =\int_{\mathbb{R}^{2}}\left|\tau_{q}^{-1} x+x_{0}\right|_{b}^{2}\left\|\varphi_{q}\right\|_{2}^{-2}\left|\varphi_{q}(x)\right|^{2} d x \\
& \rightarrow\|Q\|_{2}^{-2} \int_{\mathbb{R}^{2}}\left|x_{0}\right|_{b}^{2}|Q(x)|^{2} d x
\end{aligned}
$$

and

$$
\int_{\mathbb{R}^{2}}|x|_{b}\left|w_{q}(x)\right|^{2} d x \rightarrow\|Q\|_{2}^{-2} \int_{\mathbb{R}^{2}}\left|x_{0}\right|_{b}|Q(x)|^{2} d x
$$

as $q \rightarrow 2_{+}$. Since $t_{q}>\tilde{t}_{0}=\sqrt{\frac{q-2}{12 q}}$, we see from (4.17)-(4.19) that

$$
(q-2)^{\frac{3}{2}} \tau_{q}^{2}\left|1-t_{q}^{q-2}\right| \leq C
$$

Now, we claim that

$$
\left|1-t_{q}\right| \leq \tau_{q}^{-\frac{3}{2}}
$$

Indeed, if this is not the case, there would exist $q_{n} \rightarrow 2_{+}$such that either

$$
t_{1 q_{n}} \geq 1+\tau_{q_{n}}^{-\frac{3}{2}} \text { or } t_{1 q_{n}} \leq 1-\tau_{q_{n}}^{-\frac{3}{2}} .
$$

If $t_{1 q_{n}} \geq 1+\tau_{q_{n}}^{-\frac{3}{2}}$, then

$$
\begin{aligned}
\left(q_{n}-2\right)^{\frac{3}{2}} \tau_{q_{n}}^{2}\left|1-t_{q_{n}}^{q_{n}-2}\right| & \geq\left(q_{n}-2\right)^{\frac{3}{2}} \tau_{q_{n}}^{2}\left[\left(1+\tau_{q_{n}}^{-\frac{3}{2}}\right)^{q_{n}-2}-1\right] \\
& =\left(q_{n}-2\right)^{\frac{3}{2}} \tau_{q_{n}}^{2}\left[e^{\left(q_{n}-2\right) \log \left(1+\tau_{q_{n}}^{-\frac{3}{2}}\right)}-1\right] \\
& \geq\left(q_{n}-2\right)^{\frac{3}{2}} \tau_{q_{n}}^{2} \frac{1}{2}\left(q_{n}-2\right) \tau_{q_{n}}^{-\frac{3}{2}} \rightarrow+\infty,
\end{aligned}
$$

which contradicts (4.20). The case $t_{1 q_{n}} \leq 1-\tau_{q_{n}}^{-\frac{3}{2}}$ can be ruled out in the same way.

Next, we deal with

$$
\int_{\mathbb{R}^{2}} V(x)\left|w_{q}^{t_{q}}(x)\right|^{2} d x
$$

It holds

$$
\begin{aligned}
\int_{\mathbb{R}^{2}} V(x)\left|w_{q}^{t_{q}}(x)\right|^{2} d x & =\int_{\mathbb{R}^{2}}\left(\left|\frac{x}{t_{q} \tau_{q}}+\frac{x_{0}}{t_{q}}\right|_{b}-\left|x_{0}\right| b\right)^{2} \frac{\left|\varphi_{q}(x)\right|^{2}}{\left\|\varphi_{q}\right\|_{2}^{2}} d x \\
& =\frac{1}{\tau_{q}^{2}} \int_{\mathbb{R}^{2}}\left(\left|\frac{x}{t_{q}}+\frac{x_{0} \tau_{q}}{t_{q}}\right|_{b}-\left|\frac{x_{0} \tau_{q}}{t_{q}}\right|_{b}+\left|\frac{x_{0} \tau_{q}}{t_{q}}\right|_{b}-\left|x_{0} \tau_{q}\right|_{b}\right)^{2} \frac{\left|\varphi_{q}(x)\right|^{2}}{\left\|\varphi_{q}\right\|_{2}^{2}} d x .
\end{aligned}
$$


By (4.21) and $\left|x_{0}\right|_{b}=A$, we get

$$
\begin{aligned}
\left|\frac{x}{t_{q}}+\frac{x_{0} \tau_{q}}{t_{q}}\right|_{b}-\left|\frac{x_{0} \tau_{q}}{t_{q}}\right|_{b} & =\frac{1}{t_{q}}\left(\left|x+x_{0} \tau_{q}\right|_{b}-\left|x_{0} \tau_{q}\right|_{b}\right) \\
& =\frac{1}{t_{q}} \frac{|x|_{b}^{2}+2\left(b_{1}^{-2} x_{1}, b_{2}^{-2} x_{2}\right) \cdot x_{0} \tau_{q}}{\left|x+x_{0} \tau_{q}\right|_{b}+\left|x_{0} \tau_{q}\right|_{b}} \\
& \rightarrow \frac{\left(b_{1}^{-2} x_{1}, b_{2}^{-2} x_{2}\right) \cdot x_{0}}{A}
\end{aligned}
$$

and

$$
\left.|| \frac{x_{0} \tau_{q}}{t_{q}}\right|_{b}-\left.\left|x_{0} \tau_{q}\right|\right|_{b} \leq\left|x_{0} \tau_{q}\right| b \frac{\left|1-t_{q}\right|}{\left|t_{q}\right|} \leq C \tau_{q}^{-\frac{1}{2}} \rightarrow 0
$$

as $q \rightarrow 2$. Since $Q$ is radially symmetric about the origin, by Lemma 2.1, (4.23), (4.24) and the Lebesgue dominated theorem, we have

$$
\begin{aligned}
\int_{\mathbb{R}^{2}} V(x)\left|w_{q}^{t_{q}}(x)\right|^{2} d x & =\tau_{q}^{-2}\left(\int_{\mathbb{R}^{2}} \frac{\left|b_{1}^{-2} x_{1} x_{0}^{1}\right|^{2}}{A^{2}} \frac{|Q(x)|^{2}}{\|Q\|_{2}^{2}} d x+o(1)\right) \\
& =\tau_{q}^{-2}\left(\frac{1}{2 b_{1}^{2}} \int_{\mathbb{R}^{2}} \frac{|x|^{2}|Q(x)|^{2}}{\|Q\|_{2}^{2}} d x+o(1)\right) .
\end{aligned}
$$

We derive from (4.2), (4.3), (4.24) and (4.25) that

$$
\begin{aligned}
\max _{\tilde{t}_{0} \leq t \leq \tilde{t}_{1}} E_{a, q}\left(w_{q}^{t}\right) & =E_{a, q}\left(w_{q}^{t_{q}}\right) \\
& =\frac{1}{2} \tau_{q}^{2} t_{q}^{2}-\frac{1}{q} \tau_{q}^{2} t_{q}^{q}+\int_{\mathbb{R}^{2}} V(x)\left|w_{q}^{t_{q}}(x)\right|^{2} d x \\
& \leq \tau_{q}^{2} \max _{t>0}\left\{\frac{1}{2} t^{2}-\frac{1}{q} t^{q}\right\}+\int_{\mathbb{R}^{2}} V(x)\left|w_{q}^{t_{q}}(x)\right|^{2} d x \\
& \leq \frac{q-2}{2 q} \tau_{q}^{2}+\tau_{q}^{-2}\left(\frac{1}{2 b_{1}^{2}} \int_{\mathbb{R}^{2}} \frac{|x|^{2}|Q(x)|^{2}}{\|Q\|_{2}^{2}} d x+o(1)\right),
\end{aligned}
$$

which and (4.15) immediately yield the conclusion.

Proposition 4.2. There holds

$$
C_{1}(q-2) \tau_{q}^{2} \leq \int_{\mathbb{R}^{2}}\left|\nabla u_{q}(x)\right|^{2} d x \leq \tau_{q}^{2}+\frac{C_{2}}{q-2}
$$

with $C_{1}>0, C_{2}>0$.

Proof. We deduce from Proposition 4.1, (3.21) and (3.27) that

$$
(q-2) \int_{\mathbb{R}^{2}}\left|\nabla u_{q}(x)\right|^{2} d x+\int_{\mathbb{R}^{2}}\left[q\left(|x|_{b}-A\right)^{2}+2|x|_{b}\left(|x|_{b}-A\right)\right]\left|u_{q}(x)\right|^{2} d x=(q-2) \tau_{q}^{2}+o(1),
$$

which implies

$$
\int_{\mathbb{R}^{2}}\left|\nabla u_{q}(x)\right|^{2} d x \leq \tau_{q}^{2}+\frac{C}{q-2}
$$


since $\left\|u_{q}\right\|_{2}^{2}=1$.

Now, we derive the lower bound in (4.26).

Suppose on the contrary that there exists $q_{k} \rightarrow 2_{+}$such that

$$
\int_{\mathbb{R}^{2}}\left|\nabla u_{q_{k}}(x)\right|^{2} d x=o\left(\left(q_{k}-2\right) \tau_{q_{k}}^{2}\right) \text { as } k \rightarrow \infty .
$$

By (4.27), we have

$$
\int_{\mathbb{R}^{2}}\left[q_{k}\left(|x|_{b}-A\right)^{2}+|x|_{b}\left(|x|_{b}-A\right)\right]\left|u_{q_{k}}(x)\right|^{2} d x=O\left(\left(q_{k}-2\right) \tau_{q_{k}}^{2}\right) .
$$

Observing that

$$
q\left(|x|_{b}-A\right)^{2}+|x|_{b}\left(|x|_{b}-A\right) \leq(q+1)|x|_{b}^{2}+q A^{2}
$$

and $\left\|u_{q}\right\|_{2}^{2}=1$, we have

$$
\int_{\mathbb{R}^{2}}|x|_{b}^{2}\left|u_{q_{k}}(x)\right|^{2} d x \geq O\left(\left(q_{k}-2\right) \tau_{q_{k}}^{2}\right)
$$

Therefore,

$$
\begin{aligned}
\int_{\mathbb{R}^{2}}|x|_{b}\left(|x|_{b}-A\right)\left|u_{q_{k}}(x)\right|^{2} d x & \geq \int_{\mathbb{R}^{2}}\left(\frac{1}{2}|x|_{b}^{2}-\frac{1}{2} A^{2}\right)\left|u_{q_{k}}(x)\right|^{2} d x \\
& \geq O\left(\left(q_{k}-2\right) \tau_{q_{k}}^{2}\right) .
\end{aligned}
$$

By (3.27) we obtain

$$
\int_{\mathbb{R}^{2}}\left|\nabla u_{q_{k}}(x)\right|^{2} \geq \int_{\mathbb{R}^{2}}|x|_{b}\left(|x|_{b}-A\right)\left|u_{q_{k}}(x)\right|^{2} d x \geq O\left(\left(q_{k}-2\right) \tau_{q_{k}}^{2}\right),
$$

a contradiction.

Proof of Theorem 1.2. It is known from (4.27) that

$$
\int_{\mathbb{R}^{2}}\left[q\left(|x|_{b}-A\right)^{2}+|x|_{b}\left(|x|_{b}-A\right)\right]\left|u_{q}(x)\right|^{2} d x \leq C(q-2) \tau_{q}^{2} .
$$

Then, the fact that

$$
|x|_{b}\left(|x|_{b}-A\right) \geq \frac{1}{2}\left(|x|_{b}^{2}-A^{2}\right)
$$

and $\left\|u_{q}\right\|_{2}^{2}=1$ yield

$$
\int_{\mathbb{R}^{2}}|x|_{b}^{2}\left|u_{q}(x)\right|^{2} d x \leq C(q-2) \tau_{q}^{2}
$$

Therefore,

$$
\int_{\mathbb{R}^{2}}\left(|x|_{b}-A\right)^{2}\left|u_{q}(x)\right|^{2} d x \leq 2 \int_{\mathbb{R}^{2}}\left(|x|_{b}^{2}+A^{2}\right)\left|u_{q}(x)\right|^{2} d x \leq C(q-2) \tau_{q}^{2}
$$

and

$$
\left.\left.\left|\int_{\mathbb{R}^{2}}\right| x\right|_{b}\left(|x|_{b}-A\right)\left|u_{q}(x)\right|^{2} d x\left|\leq \int_{\mathbb{R}^{2}} \frac{1}{2}\left(3|x|_{b}^{2}+A^{2}\right)\right| u_{q}(x)\right|^{2} d x \leq C(q-2) \tau_{q}^{2} .
$$


Since $-\Delta+V$ is a compact operator, its first eigenvalue $\lambda_{1}$ and corresponding eigenfunction $\varphi_{1}$ are positive, which satisfy

$$
-\Delta \varphi+V \varphi=\lambda_{1} \varphi
$$

Hence, by (3.26),

$$
\begin{aligned}
\int_{\mathbb{R}^{2}} \mu_{q} u_{q} \varphi_{1} d x & <\int_{\mathbb{R}^{2}}\left(-\Delta u_{q}+V u_{q}\right) \varphi_{1} d x \\
& =\int_{\mathbb{R}^{2}}\left(-\Delta \varphi_{1}+V \varphi_{1}\right) u_{q} d x \\
& =\int_{\mathbb{R}^{2}} \lambda_{1} \varphi_{1} u_{q} d x
\end{aligned}
$$

implying

$$
\mu_{q}<\lambda_{1}
$$

Using (3.26) again, we get

$$
\mu_{q}=\int_{\mathbb{R}^{2}}\left|\nabla u_{q}(x)\right|^{2} d x+\int_{\mathbb{R}^{2}} V(x)\left|u_{q}(x)\right|^{2} d x-a \int_{\mathbb{R}^{2}}\left|u_{q}(x)\right|^{q+2} d x .
$$

Equation (4.30) and Proposition 4.2 yield for $q>2$ and close to 2 that

$$
a \int_{\mathbb{R}^{2}}\left|u_{q}(x)\right|^{q+2} d x \geq \int_{\mathbb{R}^{2}}\left|\nabla u_{q}(x)\right|^{2} d x-\lambda_{1} \geq \frac{1}{2} \int_{\mathbb{R}^{2}}\left|\nabla u_{q}(x)\right|^{2} d x .
$$

By (3.27), Proposition 4.2 and (4.29),

$$
\begin{aligned}
\frac{q a}{q+2} \int_{\mathbb{R}^{2}}\left|u_{q}(x)\right|^{q+2} d x & \leq \int_{\mathbb{R}^{2}}\left|\nabla u_{q}(x)\right|^{2} d x+\left.\left|\int_{\mathbb{R}^{2}}\right| x\right|_{b}\left(|x|_{b}-A\right)\left|u_{q}(x)\right|^{2} d x \mid \\
& \leq C \int_{\mathbb{R}^{2}}\left|\nabla u_{q}(x)\right|^{2} d x .
\end{aligned}
$$

Let $\varepsilon_{q}=\left\|\nabla u_{q}\right\|_{2}^{-1}$ and $\tilde{w}_{q}(x)=\varepsilon_{q} u_{q}\left(\varepsilon_{q} x\right)$. Then, $\left\|\nabla \tilde{w}_{q}\right\|_{2}^{2}=\left\|\tilde{w}_{q}\right\|_{2}^{2}=1$. We know from Lemma 2.1 that $a_{q}^{*} \rightarrow a^{*}$ as $q \rightarrow 2_{+}$, and then

$$
\begin{aligned}
\varepsilon_{q}^{q-2} & =\left(\int_{\mathbb{R}^{2}}\left|\nabla u_{q}(x)\right|^{2} d x\right)^{\frac{2-q}{2}} \\
& \geq C^{\frac{2-q}{2}} \tau_{q}^{2-q} \\
& =C^{\frac{2-q}{2}} \frac{q a}{2 a_{q}^{*}} \rightarrow \frac{a}{a^{*}}
\end{aligned}
$$

and

Thus

$$
\varepsilon_{q}^{q-2} \leq C^{\frac{2-q}{2}}(q-2)^{\frac{2-q}{2}} \tau_{q}^{2-q} \rightarrow \frac{a}{a^{*}}
$$

$$
\varepsilon_{q}^{q-2} \rightarrow \frac{a}{a^{*}} \text { as } q \rightarrow 2_{+}
$$

It follows from (4.32)-(4.34) that there exist $C_{1}>0$ and $C_{2}>0$ such that

$$
C_{1} \leq \int_{\mathbb{R}^{2}}\left|\tilde{w}_{q}(x)\right|^{q+2} d x=\varepsilon_{q}^{q-2} \frac{\int_{\mathbb{R}^{2}}\left|u_{q}(x)\right|^{q+2} d x}{\int_{\mathbb{R}^{2}}\left|\nabla u_{q}(x)\right|^{2} d x} \leq C_{2} .
$$


Now we claim that there exist $\left\{y_{q}\right\} \subset \mathbb{R}^{2}, R_{0}>0$ and $\eta>0$ such that

$$
\liminf _{q \rightarrow 2+} \int_{B_{R_{0}}\left(y_{q}\right)}\left|\tilde{w}_{q}(x)\right|^{2} d x \geq \eta
$$

Indeed, if this is not the case, for any $R>0$, there exists a sequence $\left\{\tilde{w}_{q_{k}}\right\}$ with $q_{k} \rightarrow 2_{+}$ as $k \rightarrow \infty$ such that

$$
\lim _{k \rightarrow \infty} \sup _{x \in \mathbb{R}^{2}} \int_{B_{R}(y)}\left|\tilde{w}_{q}(x)\right|^{2} d x=0 .
$$

By Lemma 1.21 in [22], we get $\tilde{w}_{q_{k}} \rightarrow 0$ strongly in $L^{\gamma}\left(\mathbb{R}^{2}\right)$ for any $\gamma>2$, which contradicts to $(4.35)$.

Denote $w_{q}(x)=\tilde{w}_{q}\left(x+y_{q}\right)$. Then

$$
\begin{gathered}
\left\|\nabla w_{q}\right\|_{2}^{2}=1 \\
C_{1} \leq \int_{\mathbb{R}^{2}}\left|w_{q}(x)\right|^{q+2} d x \leq C_{2} ; \\
\liminf _{q \rightarrow 2_{+}} \int_{B_{R_{0}}(0)}\left|w_{q}(x)\right|^{2} d x \geq \eta .
\end{gathered}
$$

Hence, there exist a sequence $\left\{q_{k}\right\}, q_{k} \rightarrow 2_{+}$as $k \rightarrow \infty$, and $w \in H^{1}\left(\mathbb{R}^{2}\right)$ such that $w_{q_{k}}:=w_{k} \rightarrow w$ weakly in $\mathcal{H}$ and $w_{k} \rightarrow w \neq 0$ strongly in $L_{l o c}^{\gamma}\left(\mathbb{R}^{2}\right)$ for any $\gamma>2$. Denote $\varepsilon_{q_{k}}:=\varepsilon_{k}$ and $y_{q_{k}}=y_{k}$ for simiplity. By (3.26), we find that $w_{k}$ solves

$$
-\Delta w_{k}+\varepsilon_{k}^{2}\left(\left|\varepsilon_{k}\left(x+y_{k}\right)\right|_{b}-A\right)^{2} w_{k}=\varepsilon_{k}^{2} \mu_{q_{k}} w_{k}+\varepsilon_{k}^{2-q_{k}} a w_{k}^{q_{k}+1} .
$$

Now, we study the asymptotic behavior of $w_{k}$. By (4.30), the definition of $\varepsilon_{k}$ and Proposition 4.2 , we have

$$
\varepsilon_{k}^{2} \mu_{q_{k}} \leq \varepsilon_{k}^{2} \lambda_{1} \rightarrow 0 \text { as } k \rightarrow \infty .
$$

It follows from (4.31) and (4.32) that

$$
\varepsilon_{k}^{2} \mu_{q_{k}} \geq-\varepsilon_{k}^{2} a \int_{\mathbb{R}^{2}}\left|u_{q_{k}}(x)\right|^{q_{k}+2} d x=-\frac{a \int_{\mathbb{R}^{2}}\left|u_{q_{k}}(x)\right|^{q_{k}+2} d x}{\int_{\mathbb{R}^{2}}\left|\nabla u_{q_{k}}(x)\right|^{2} d x} \geq-C .
$$

So we may assume

$$
\varepsilon_{k}^{2} \mu_{q_{k}} \rightarrow-\beta^{2} \leq 0
$$

Next, we study the asymptotic behavior of $\varepsilon_{k}^{2}\left(\left|\varepsilon_{k}\left(x+y_{k}\right)\right|_{b}-A\right)^{2}$.

If $\liminf _{k \rightarrow \infty}\left|\varepsilon_{k} y_{k}\right|_{b} \leq C$, there exists a subsequence of $\left\{q_{k}\right\}$, still denoted by $\left\{q_{k}\right\}$, such that $\left|\varepsilon_{k} y_{k}\right|_{b} \leq C$, so for any $M>0$, we have

$$
\varepsilon_{k}^{2}\left(\left|\varepsilon_{k}\left(x+y_{k}\right)\right|_{b}-A\right)^{2} \rightarrow 0
$$

uniformly for $x \in B_{M}(0)$ as $k \rightarrow \infty$.

If $\liminf _{k \rightarrow \infty}\left|\varepsilon_{k} y_{k}\right|_{b}=\infty$, there exists a subsequence of $\left\{q_{k}\right\}$, still denoted by $\left\{q_{k}\right\}$, such that $\left|\varepsilon_{k} y_{k}\right|_{b} \rightarrow \infty$, as $k \rightarrow \infty$. For any $M>0$, if $|x|_{b} \leq M$ and $k$ is large enough, we obtain

$$
\left(\left|\varepsilon_{k}\left(x+y_{k}\right)\right|_{b}-A\right)^{2} \geq \frac{1}{2}\left|\varepsilon_{k} y_{k}\right|_{b}^{2} .
$$


Equation (4.28) then implies that

$$
\begin{aligned}
\int_{B_{R_{0}}(0)} \frac{1}{2}\left|\varepsilon_{k} y_{k}\right|_{b}^{2}\left|w_{k}(x)\right|^{2} d x & \leq \int_{\mathbb{R}^{2}}\left(\left|\varepsilon_{k}\left(x+y_{k}\right)\right|_{b}-A\right)^{2}\left|w_{k}(x)\right|^{2} d x \\
& =\int_{\mathbb{R}^{2}}\left(|x|_{b}-A\right)^{2}\left|u_{q_{k}}(x)\right|^{2} d x \\
& \leq C\left(q_{k}-2\right) \tau_{q_{k}}^{2} .
\end{aligned}
$$

Consequently, by (4.37), we have

$$
\left|\varepsilon_{k} y_{k}\right|_{b}^{2} \leq C\left(q_{k}-2\right) \tau_{q_{k}}^{2} .
$$

Let $\varepsilon_{q}=\left\|\nabla u_{q}\right\|_{2}^{-1}$. By Proposition 4.2 ,

$$
\varepsilon_{k}^{2}\left|\varepsilon_{k} y_{k}\right|_{b}^{2} \leq \frac{C\left(q_{k}-2\right) \tau_{q_{k}}^{2}}{\int_{\mathbb{R}^{2}}\left|\nabla u_{q_{k}}(x)\right|^{2} d x} \leq C .
$$

We may assume that $\varepsilon_{k}^{2}\left|\varepsilon_{k} y_{k}\right|_{b}^{2} \rightarrow \mu \geq 0$. It is easy to show that for any $M>0$ and $x \in B_{M}(0)$,

$$
\lim _{k \rightarrow \infty} \varepsilon_{k}^{2}\left(\left|\varepsilon_{k}\left(x+y_{k}\right)\right|_{b}-A\right)^{2}=\lim _{k \rightarrow \infty} \varepsilon_{k}^{2}\left|\varepsilon_{k} y_{k}\right|_{b}^{2}=\mu \geq 0 .
$$

In summary of (4.34) and (4.41)-(4.44), we have the following cases.

Case (i): $\varepsilon_{k} \mu_{q_{k}} \rightarrow 0$, and $\varepsilon_{k}^{2}\left(\left|\varepsilon_{k}\left(x+y_{k}\right)\right|_{b}-A\right)^{2} \rightarrow 0$;

Case (ii): $\varepsilon_{k} \mu_{q_{k}} \rightarrow-\beta^{2}<0$, and $\varepsilon_{k}^{2}\left(\left|\varepsilon_{k}\left(x+y_{k}\right)\right|_{b}-A\right)^{2} \rightarrow 0$;

Case (iii): $\varepsilon_{k} \mu_{q_{k}} \rightarrow 0$, and $\varepsilon_{k}^{2}\left(\left|\varepsilon_{k}\left(x+y_{k}\right)\right|_{b}-A\right)^{2} \rightarrow \mu>0$;

Case (iv): $\varepsilon_{k} \mu_{q_{k}} \rightarrow-\beta^{2}<0$, and $\varepsilon_{k}^{2}\left(\left|\varepsilon_{k}\left(x+y_{k}\right)\right|_{b}-A\right)^{2} \rightarrow \mu>0$;

Taking the limit as $k \rightarrow \infty$ in (4.40), we obtain that $w$ satisfies correspondingly in the case (i) that

$$
-\Delta w=a^{*} w^{3}
$$

in the case (ii) that

$$
-\Delta w+\beta^{2} w=a^{*} w^{3}
$$

in the case (iii) that

$$
-\Delta w+\mu w=a^{*} w^{3}
$$

in the case (iv) that

$$
-\Delta w+\left(\mu+\beta^{2}\right) w=a^{*} w^{3} .
$$

The case (i) can not happen. Indeed, if it would happen on the contrary, the fact that $w \geq 0$ and $w \neq 0$ would imply that the equation (4.44) admits a positive solution, which contradicts the Liouville theorem.

In the case (ii), by the uniqueness of positive solution of (4.45) and $w \neq 0$, there exists $y_{0} \in \mathbb{R}^{2}$ such that

$$
w=\frac{\beta}{\|Q\|_{2}} Q\left(\beta\left(x-y_{0}\right)\right),
$$

which implies $\|w\|_{2}^{2}=1$. Hence $w_{q_{k}} \rightarrow w$ strongly in $L^{2}\left(\mathbb{R}^{2}\right)$, that is

$$
\varepsilon_{k} u_{q_{k}}\left(\varepsilon_{k}\left(x+y_{k}\right)\right) \rightarrow \frac{\beta}{\|Q\|_{2}} Q\left(\beta\left(x-y_{0}\right)\right) \text { strongly in } L^{2}\left(\mathbb{R}^{2}\right) \text {. }
$$


Let $x_{k}=\varepsilon_{k}\left(y_{k}+y_{0}\right)$. Then

$$
\bar{w}_{k}:=\varepsilon_{k} u_{q_{k}}\left(\varepsilon_{k} x+x_{k}\right) \rightarrow \frac{\beta}{\|Q\|_{2}} Q(\beta x) \text { strongly in } L^{2}\left(\mathbb{R}^{2}\right) .
$$

The case (iii) and (iv) can be treated in the same way as the case (ii), we omit the detail. The proof is complete.

Finally, we study the limiting behavior of $x_{k}$.

Proof of Theorem 1.3 It is obvious that either $\liminf _{k \rightarrow \infty}\left|x_{k}\right|=+\infty$ or $\liminf _{k \rightarrow \infty}\left|x_{k}\right|$ is bounded.

If $\liminf \operatorname{in}_{k \rightarrow \infty}\left|x_{k}\right|=+\infty$, there is nothing to prove.

If $\liminf _{k \rightarrow \infty}\left|x_{k}\right|$ is bounded, there exist $x_{0} \in \mathbb{R}^{2}$ and a subsequence of $\left\{x_{k}\right\}$, still denoted by $\left\{x_{k}\right\}$ such that $x_{k} \rightarrow x_{0}$, as $k \rightarrow \infty$. We may show as the proof of Theorem 1.2 that only the case (ii) may happen, that is, $\varepsilon_{k}^{2} \mu_{q_{k}} \rightarrow-\beta^{2}<0$ and $\varepsilon_{k}^{2-q_{k}} a \rightarrow a^{*}$. By (4.40), we have

$$
-\Delta w_{q_{k}} \leq \varepsilon_{k}^{2-q_{k}} a w_{k}^{q_{k}+1}
$$

Using the De Diorgi-Nash-Moser estimate, see Theorem 4.1 in [17], we obtain

$$
\begin{aligned}
\max _{x \in B_{1}(\xi)} w_{k}(x) & \leq C\left(\int_{B_{2}(\xi)}\left|w_{k}(x)\right|^{2} d x\right)^{\frac{1}{2}} \\
& \leq C\left[\left(\int_{B_{2}(\xi)}\left|w_{k}(x)-w\right|^{2} d x\right)^{\frac{1}{2}}+\left(\int_{B_{2}(\xi)}|w(x)|^{2} d x\right)^{\frac{1}{2}}\right] .
\end{aligned}
$$

Since $w_{q_{k}} \rightarrow w$ strongly in $L^{2}\left(\mathbb{R}^{2}\right), w_{k}$ is uniformly bounded in $L^{\infty}\left(\mathbb{R}^{2}\right)$. Hence, there exists $R>0$ such that if $k$ is large enough and $|x| \geq R$,

$$
\varepsilon_{k}^{2-q_{k}} a w_{k}^{q_{k}} \leq \frac{\beta^{2}}{2}
$$

and

$$
-\Delta w_{k} \leq-\frac{1}{3} \beta^{2} w_{k}
$$

By the comparison principle,

$$
w_{k} \leq C e^{-\frac{1}{4} \beta^{2}|x|} \text { for }|x| \geq R .
$$

Since $w_{k}$ is uniformly bounded in $L^{\infty}\left(\mathbb{R}^{2}\right)$, we have

$$
w_{k} \leq C e^{-\frac{1}{4} \beta^{2}|x|} \text { for any } x \in \mathbb{R}^{2} .
$$

Noting $x_{k}=\varepsilon_{k}\left(y_{k}+y_{0}\right)$ is bounded in $\mathbb{R}^{2}$, we have

$$
\int_{\mathbb{R}^{2}}\left(|x|_{b}-A\right)^{2}\left|u_{q_{k}}(x)\right|^{2} d x=\int_{\mathbb{R}^{2}}\left(\left|\varepsilon_{k}\left(x+y_{k}\right)\right|_{b}-A\right)^{2}\left|w_{k}(x)\right|^{2} d x \leq C .
$$

Similarly,

$$
\left.\left|\int_{\mathbb{R}^{2}}\right| x\right|_{b}\left(|x|_{b}-A\right)\left|u_{q_{k}}(x)\right|^{2} d x \mid \leq C \quad \text { and } \quad \int_{\mathbb{R}^{2}}|x|_{b}^{2}\left|u_{q_{k}}(x)\right|^{2} d x \leq C .
$$


By (4.27), (4.48) and (4.49), we obtain

$$
\lim _{k \rightarrow \infty} \tau_{k}^{-2} \int_{\mathbb{R}^{2}}\left|\nabla u_{q_{k}}\right|^{2} d x=1
$$

Let

$$
\begin{aligned}
g(t) & =: E_{a, q_{k}}\left(u_{q_{k}}^{t}\right) \\
& =\frac{1}{2} t^{2} \int_{\mathbb{R}^{2}}\left|\nabla u_{q_{k}}\right|^{2} d x-\frac{a t^{q_{k}}}{q_{k}+2} \int_{\mathbb{R}^{2}}\left|u_{q_{k}}(x)\right|^{q_{k}+2} d x \\
& +\frac{1}{2} t^{-2} \int_{\mathbb{R}^{2}}|x|_{b}^{2}\left|u_{q_{k}}(x)\right|^{2} d x-A t^{-1} \int_{\mathbb{R}^{2}}|x|_{b}\left|u_{q_{k}}(x)\right|^{2} d x+\frac{1}{2} A^{2} \int_{\mathbb{R}^{2}}\left|u_{q_{k}}(x)\right|^{2} d x \\
& :=\frac{1}{2} B t^{2}-\frac{1}{q} C t^{q_{k}}+\frac{1}{2} D t^{-2}-E t^{-1}+F .
\end{aligned}
$$

Then

$$
\begin{aligned}
g^{\prime}(t) & =B t-C t^{q_{k}-1}-D t^{-3}+E t^{-2} \\
& =t^{-3}\left(B t^{4}-C t^{q_{k}+2}-D+E t\right) \\
& :=t^{-3} h(t), \\
h^{\prime}(t) & =4 B t^{3}-C\left(q_{k}+2\right) t^{q_{k}+1}+E,
\end{aligned}
$$

and

$$
h^{\prime \prime}(t)=12 B t^{2}-C\left(q_{k}+2\right)\left(q_{k}+1\right) t^{q_{k}} .
$$

It is easy to deduce that there exist $t_{1}>0$ and $t_{2}>0$ such that

(i) $g(t)$ is decreasing in $\left(0, t_{1}\right)$ and $\left(t_{2},+\infty\right)$, while $g(t)$ is increasing in $\left(t_{1}, t_{2}\right)$;

(ii) $g^{\prime}\left(t_{1}\right)=g^{\prime}\left(t_{2}\right)=0$ and $\lim _{t \rightarrow 0} g(t)=\infty$.

In order to estimate $t_{1}$ and $t_{2}$, we rewrite $g^{\prime}(t)$ as

$$
\begin{aligned}
g^{\prime}(t) & =t \int_{\mathbb{R}^{2}}\left|\nabla u_{q_{k}}\right|^{2} d x-\frac{q_{k} a}{q_{k}+2} t^{q_{k}-1} \int_{\mathbb{R}^{2}}\left|u_{q_{k}}(x)\right|^{q_{k}+2} d x \\
& -\frac{1}{t^{3}} \int_{\mathbb{R}^{2}}|x|_{b}^{2}\left|u_{q_{k}}(x)\right|^{2} d x+A t^{-2} \int_{\mathbb{R}^{2}}|x|_{b}\left|u_{q_{k}}(x)\right|^{2} d x .
\end{aligned}
$$

By the Pohozaev identity (3.27), we have $g^{\prime}(1)=0$.

Now, we claim that $t_{2}=1$. Indeed, we choose $t_{3}=C\left(q_{k}-2\right)^{\frac{1}{2}}$ such that

$$
\frac{1}{2} \int_{\mathbb{R}^{2}}\left|\nabla u_{q_{k}}^{t_{3}}(x)\right|^{2} d x \leq \frac{q_{k}-2}{4 q_{k}} \tau_{k}^{2} .
$$

Then by (4.49), we have

$$
\begin{aligned}
\frac{1}{2} \int_{\mathbb{R}^{2}} V(x)\left|u_{q_{k}}^{t_{3}}(x)\right|^{2} d x & =\frac{1}{2} \int_{\mathbb{R}^{2}}\left(t_{3}^{-1}|x|_{b}-A\right)^{2}\left|u_{q_{k}}(x)\right|^{2} d x \\
& \leq \int_{\mathbb{R}^{2}}\left(t_{3}^{-2}|x|_{b}^{2}+A^{2}\right)\left|u_{q_{k}}(x)\right|^{2} d x \\
& \leq C\left(q_{k}-2\right)^{-2} .
\end{aligned}
$$


By Proposition 4.2, for $q>2$ and $q$ close to 2 we have,

$$
\begin{aligned}
g\left(t_{3}\right) & \leq \frac{1}{2}\left(\int_{\mathbb{R}^{2}}\left|\nabla u_{q_{k}}^{t_{3}}(x)\right|^{2} d x+\int_{\mathbb{R}^{2}} V(x)\left|u_{q_{k}}^{t_{3}}(x)\right|^{2} d x\right) \\
& \leq \frac{q_{k}-2}{4 q_{k}} \tau_{k}^{2}+C\left(q_{k}-2\right)^{-2} \\
& \leq \frac{q_{k}-2}{2 q_{k}} \tau_{k}^{2}+o(1) \\
& =E_{a, q_{k}}\left(u_{q_{k}}\right)=g(1) .
\end{aligned}
$$

Hence, $t_{2}=1$ and

$$
g(1)=E_{a, q_{k}}\left(u_{q_{k}}\right) \geq g(t)=E_{a, q_{k}}\left(u_{q_{k}}^{t}\right) \text { for any } t \in\left(C\left(q_{k}-2\right)^{\frac{1}{2}},+\infty\right) .
$$

Choose $t_{k}$ such that

$$
\tilde{Q}_{q_{k}}\left(u_{q_{k}}^{t_{k}}\right)=t_{k}^{2} \int_{\mathbb{R}^{2}}\left|\nabla u_{q_{k}}(x)\right|^{2} d x-\frac{q_{k} a}{q_{k}+2} t_{k}^{q_{k}} \int_{\mathbb{R}^{2}}\left|u_{q_{k}}(x)\right|^{q_{k}+2} d x=0 .
$$

By (3.27), (4.49) and (4.50), we obtain

$$
\begin{aligned}
t_{k} & =\left[\frac{\left(q_{k}+2\right) \int_{\mathbb{R}^{2}}\left|\nabla u_{q_{k}}(x)\right|^{2} d x}{q_{k} a \int_{\mathbb{R}^{2}}\left|u_{q_{k}}(x)\right|^{q_{k}+2} d x}\right]^{\frac{1}{q_{k}-2}} \\
& =\left[\frac{\int_{\mathbb{R}^{2}}\left|\nabla u_{q_{k}}(x)\right|^{2} d x}{\int_{\mathbb{R}^{2}}\left|\nabla u_{q_{k}}(x)\right|^{2} d x-\int_{\mathbb{R}^{2}}|x|_{b}\left(|x|_{b}-A\right)\left|u_{q_{k}}(x)\right|^{2} d x}\right]^{\frac{1}{q_{k}-2}} \\
& =\left[1+O\left(\tau_{k}^{-2}\right)\right]^{\frac{1}{q_{k}-2}} .
\end{aligned}
$$

Therefore,

$$
\left|t_{k}-1\right|=\left|e^{\frac{1}{q_{k}-2} \log \left(1+O\left(\tau_{k}^{-2}\right)\right)}-1\right|=\frac{1}{q_{k}-2} O\left(\tau_{k}^{-2}\right) \text { as } k \rightarrow \infty
$$

and $t_{k}>t_{3}$ for $k$ large enough.

By Lemma 2.2 and Remark 2.1, we get

$$
\left.\inf _{g \in \tilde{\Gamma}_{q}} \max _{t \in[0,1]} E_{a, q}\right|_{V=0}(g(t))=\inf \left\{\left.E_{a, q}\right|_{V=0}(u): \tilde{Q}_{q}(u)=0, u \in \tilde{S}(1)\right\}=\frac{q-2}{2 q} \tau_{q}^{2} .
$$

We derive from (4.51) and (4.53) that

$$
\begin{aligned}
g(1) & =E_{a, q_{k}}\left(u_{q_{k}}\right) \\
& \geq g\left(t_{k}\right) \\
& =E_{a, q_{k}}\left(u_{q_{k}}^{t_{k}}\right) \\
& =\left.E_{a, q_{k}}\right|_{V=0}\left(u_{q_{k}}^{t_{k}}\right)+\int_{\mathbb{R}^{2}} V(x)\left|u_{q_{k}}^{t_{k}}(x)\right|^{2} d x \\
& \geq \inf \left\{\left.E_{a, q}\right|_{V=0}(u): \tilde{Q}_{q}(u)=0, u \in \tilde{S}(1)\right\}+\int_{\mathbb{R}^{2}} V(x)\left|u_{q_{k}}^{t_{k}}(x)\right|^{2} d x \\
& =\frac{q_{k}-2}{2 q_{k}} \tau_{k}^{2}+\int_{\mathbb{R}^{2}} V(x)\left|u_{q_{k}}^{t_{k}}(x)\right|^{2} d x .
\end{aligned}
$$


Then, Proposition 4.1 yields

$$
\limsup _{k \rightarrow \infty} \tau_{k}^{2} \int_{\mathbb{R}^{2}} V(x)\left|u_{q_{k}}^{t_{k}}(x)\right|^{2} d x \leq \frac{1}{2\|Q\|_{2}^{2}} \int_{\mathbb{R}^{2}}|x|^{2}|Q(x)|^{2} d x .
$$

Next we claim that

$$
\liminf _{k \rightarrow \infty}\left|\frac{\left|x_{k}\right|_{b}-A t_{k}}{\varepsilon_{k} t_{k}}\right| \leq C .
$$

We argue by contradiction. Suppose this is not the case, there would exist a subsequence of $\left\{q_{k}\right\}$, still denoted by $\left\{q_{k}\right\}$, such that

$$
\frac{\left.|| x_{k}\right|_{b}-A t_{k} \mid}{\varepsilon_{k} t_{k}} \rightarrow \infty
$$

By the definition $\varepsilon_{k},(4.39)$ and (4.50), for any $C \geq 0$ and $k$ large enough,

$$
\begin{aligned}
\tau_{k}^{2} \int_{\mathbb{R}^{2}} V(x)\left|u_{q_{k}}^{t_{k}}(x)\right|^{2} d x & \geq \frac{1}{2} \varepsilon_{k}^{-2} \int_{\mathbb{R}^{2}} V(x)\left|u_{q_{k}}^{t_{k}}(x)\right|^{2} d x \\
& =\frac{1}{2} \varepsilon_{k}^{-2} \int_{\mathbb{R}^{2}}\left(\frac{\left|\varepsilon_{k} y+x_{k}\right|}{t_{k}}-A\right)^{2}\left|\bar{w}_{k}(y)\right|^{2} d y \\
& \geq \frac{1}{2} \int_{B_{R_{0}}\left(y_{0}\right)}\left(\left|\frac{y}{t_{k}}+\frac{x_{k}}{\varepsilon_{k} t_{k}}\right|-\frac{A}{\varepsilon_{k}}\right)^{2}\left|\bar{w}_{k}(y)\right|^{2} d y \\
& \geq C,
\end{aligned}
$$

which contradicts (4.54). Hence, we may assume that

$$
\frac{\left|x_{k}\right|_{b}-A t_{k}}{\varepsilon_{k} t_{k}} \rightarrow C_{0} \text { and } x_{k} \rightarrow x_{0} \text { with }\left|x_{0}\right|_{b}=A .
$$

By the Fatou Lemma, (4.47) and (4.50), we have

$$
\begin{aligned}
& \liminf _{k \rightarrow \infty} \tau_{k}^{2} \int_{\mathbb{R}^{2}} V(x)\left|u_{q_{k}}^{t_{k}}(x)\right|^{2} d x \\
& =\liminf _{k \rightarrow \infty} \varepsilon_{k}^{-2} \int_{\mathbb{R}^{2}} V(x)\left|u_{q_{k}}^{t_{k}}(x)\right|^{2} d x \\
& =\liminf _{k \rightarrow \infty} \int_{\mathbb{R}^{2}}\left[\left(\left|\frac{y}{t_{k}}+\frac{x_{k}}{\varepsilon_{k} t_{k}}\right|_{b}-\frac{\left|x_{k}\right|_{b}}{\varepsilon_{k} t_{k}}\right)+\left(\frac{\left|x_{k}\right|_{b}}{\varepsilon_{k} t_{k}}-\frac{A}{\varepsilon_{k}}\right)\right]^{2}\left|\bar{w}_{k}(y)\right|^{2} d y \\
& =\liminf _{k \rightarrow \infty} \int_{\mathbb{R}^{2}}\left[\frac{1}{\varepsilon_{k} t_{k}\left(\left|\varepsilon_{k} y+x_{k}\right|_{b}+\left|x_{k}\right|_{b}\right)}\left[\frac{\varepsilon_{k}^{2}\left|y_{1}\right|^{2}}{b_{1}^{2}}+\frac{\varepsilon_{k}^{2}\left|y_{2}\right|^{2}}{b_{2}^{2}}+\frac{2 \varepsilon_{k} x_{k}^{1} y_{1}}{b_{1}^{2}}+\frac{2 \varepsilon_{k} x_{k}^{2} y_{2}}{b_{2}^{2}}\right]\right. \\
& \left.+\frac{\left|x_{k}\right|_{b}-A t_{k}}{\varepsilon_{k} t_{k}}\right]^{2}\left|\bar{w}_{k}(y)\right|^{2} d y \\
& \geq \int_{\mathbb{R}^{2}}\left(\frac{1}{|A|}\left(\frac{x_{0}^{1} y_{1}}{b_{1}^{2}}+\frac{x_{0}^{2} y_{2}}{b_{2}^{2}}\right)+C_{0}\right)^{2} \frac{|Q(y)|^{2}}{\|Q\|_{2}^{2}} d y .
\end{aligned}
$$


Since $Q$ is symmetric, we deduce that

$$
\begin{aligned}
& \liminf _{k \rightarrow \infty} \tau_{k}^{2} \int_{\mathbb{R}^{2}} V(x)\left|u_{q_{k}}^{t_{k}}(x)\right|^{2} d x \\
& \geq \frac{1}{A^{2}\|Q\|_{2}^{2}} \int_{\mathbb{R}^{2}}\left(\frac{\left|x_{0}^{1}\right|^{2}\left|y_{1}\right|^{2}}{b_{1}^{4}}+\frac{\left|x_{0}^{2}\right|^{2}\left|y_{2}\right|^{2}}{b_{2}^{4}}\right)|Q(y)|^{2} d y+C_{0}^{2} \\
& =\frac{1}{2 A^{2}\|Q\|_{2}^{2}}\left(\frac{\left|x_{0}^{1}\right|^{2}}{b_{1}^{4}}+\frac{\left|x_{0}^{2}\right|^{2}}{b_{2}^{4}}\right) \int_{\mathbb{R}^{2}}|y|^{2}|Q(y)|^{2} d y+C_{0}^{2} \\
& =\frac{1}{2 A^{2}\|Q\|_{2}^{2}}\left[\frac{1}{b_{1}^{2}}\left(\frac{\left|x_{0}^{1}\right|^{2}}{b_{1}^{2}}+\frac{\left|x_{0}^{2}\right|^{2}}{b_{2}^{2}}\right)+\frac{\left|x_{0}^{2}\right|^{2}}{b_{2}^{2}}\left(\frac{1}{b_{2}^{2}}-\frac{1}{b_{1}^{2}}\right)\right] \int_{\mathbb{R}^{2}}|y|^{2}|Q(y)|^{2} d y+C_{0}^{2} \\
& =\frac{1}{2 A^{2}\|Q\|_{2}^{2}}\left[\frac{1}{b_{1}^{2}} A^{2}+\frac{\left|x_{0}^{2}\right|^{2}}{b_{2}^{2}}\left(\frac{1}{b_{2}^{2}}-\frac{1}{b_{1}^{2}}\right)\right] \int_{\mathbb{R}^{2}}|y|^{2}|Q(y)|^{2} d y+C_{0}^{2} \\
& =\frac{1}{2 A^{2}\|Q\|_{2}^{2}} \int_{\mathbb{R}^{2}}|y|^{2}|Q(y)|^{2} d y+\frac{\left|x_{0}^{2}\right|^{2}}{2 A^{2}\|Q\|_{2}^{2} b_{2}^{2}}\left(\frac{1}{b_{2}^{2}}-\frac{1}{b_{1}^{2}}\right) \int_{\mathbb{R}^{2}}|y|^{2}|Q(y)|^{2} d y+C_{0}^{2} .
\end{aligned}
$$

By (4.54), this implies $x_{0}^{2}=0$ and $C_{0}=0$, and by (4.56), $x_{0} \in \mathcal{Z}$ and

$$
\frac{\left|x_{k}\right|_{b}}{\varepsilon_{k} t_{k}}-\frac{A}{\varepsilon_{k}} \rightarrow 0 \text {. }
$$

Finally, we further study the limiting behavior of $x_{k}$. Noting $\varepsilon_{q}=\left\|\nabla u_{q}\right\|_{2}^{-1}$, by (4.50), (4.52), (4.57) and $x_{k} \rightarrow x_{0}$, we get

$$
\begin{aligned}
\left.\lim _{k \rightarrow \infty} \tau_{k}|| x_{k}\right|_{b}-A \mid & =\lim _{k \rightarrow \infty} \frac{\left.|| x_{k}\right|_{b}-A \mid}{\varepsilon_{k}} \\
& \leq \lim _{k \rightarrow \infty} \frac{\left|x_{k}\right|_{b}}{\varepsilon_{k} t_{k}}\left|t_{k}-1\right|+\left.\lim _{k \rightarrow \infty} \frac{1}{\varepsilon_{k} t_{k}}|| x_{k}\right|_{b}-A t_{k} \mid \\
& \leq \lim _{k \rightarrow \infty} \frac{C\left|x_{k}\right| b}{\varepsilon_{k} t_{k}} \frac{\tau_{k}^{-2}}{q_{k}-2}+\left.\lim _{k \rightarrow \infty} \frac{1}{\varepsilon_{k} t_{k}}|| x_{k}\right|_{b}-A t_{k} \mid \\
& =\lim _{k \rightarrow \infty} \frac{C \varepsilon_{k}\left|x_{k}\right|_{b}}{t_{k}\left(q_{k}-2\right)}+\left.\lim _{k \rightarrow \infty} \frac{1}{\varepsilon_{k} t_{k}}|| x_{k}\right|_{b}-A t_{k} \mid \\
& \rightarrow 0 .
\end{aligned}
$$

The proof is complete.

Acknowledgment The first author is supported by NNSF of China, No:11671179 and 11771300. The second author is supported by NNSF of China, No:11701260.

\section{REFERENCES}

[1] A. Azzollini and A. Pomponio, On the Schrödinger equation in $R^{N}$ under the effect of a general nonlinear term, Indiana Univ. Math. J., 58 (2009), no. 3, 1361-1378.

[2] T.Bartsch and N.Soave, A natural constraint approach to normalized solutions of nonlinear Schröinger equations and systems, Journal of Functional Analysis, 272(2017), 4998-5037.

[3] T. Bartsch, S. de Valeriola, Normalized solutions of nonlinear Schröinger equations, Arch. Math., 100(1) (2012) 75-83. 
[4] J. Bellazzini, L. Jeanjean, On dipolar quantum gases in the unstable regime, SIAM J. Math. Anal., 48,(2016), 2028-2058.

[5] H. Berestycki and P. L. Lions, Nonlinear scalar field equations, I and II, Arch. Rat. Mech. Anal., 82 (1983), 313-346, 347-375.

[6] T. Cazenave, Semilinear Schrodinger Equations, Courant Lecture Notes in Mathematics Vol. 10, Courant Institute of Mathematical Science/AMS New York, 2003.

[7] F. Dalfovo, S. Giorgini, L.P. Pitaevskii, S. Stringari, Theory of Bose-Einstein condensation in trapped gases, Rev. Mod. Phys., 71,(1999), 463-512.

[8] N. Ghoussoub, Duality and Perturbation Methods in Critical Point Theory, Cambridge Tracts in Mathematics, vol. 107, Cambridge University Press, Cambridge, 1993, with appendices by David Robinson.

[9] C. Huepe, S. Metens, G. Dewel, P. Borckmans, M.E. Brachet, Decay rates in attractive Bose-Einstein condensates. Phys. Rev. Lett., 82, (1999), 1616-1619.

[10] H. Guo, H. Zhou, A constrained variational problem arising in attractive Bose-Einstein condensate with ellipse-shaped potential, Appl. Math. Lett 87(2019), 35-41.

[11] Y. J. Guo and R. Seiringer, On the mass concentration for Bose-Einstein condensates with attractive interactions, Lett. Math. Phys. 104, (2014), 141-156.

[12] Y. J. Guo, X. Y. Zeng and H. S. Zhou, Energy estimates and symmetry breaking in attractive BoseEinstein condensates with ring-shaped potentials, Ann. I. H. Poincare-AN 33 (2016), 809-828.

[13] Y. J. Guo, X. Y. Zeng and H. S. Zhou, Concentration behavior of standing waves for almost mass critical nonlinear Schrödinger equations, J. Differential Equations, 256 (2014), 2079-2100.

[14] L. Jeanjean. Existence of solutions with prescribed norm for semilinear elliptic equations, Nonlinear Anal., 28(10) (1997), 1633-1659.

[15] Y. Kagan, A.E. Muryshev, G.V. Shlyapnikov, Collapse and Bose-Einstein condensation in a trapped Bose gas with negative scattering length. Phys. Rev. Lett., 81, (1998), 933-937.

[16] M.K. Kwong, Uniqueness of positive solutions of $\nabla u-u+u^{p}=0$ in $\mathbb{R}^{N}$, Arch. Ration. Mech. Anal., 105,(1989), 243-266.

[17] Q. Han and F. H. Lin, Elliptic Partial Differential Equations: Second Edition, Courant Lecture Notes in Mathematics Vol. 1, Courant Institute of Mathematical Science/AMS, New York, 2011.

[18] P. L. Lions, The concentration-compactness principle in the caclulus of variations. The locally compact case II, Ann. Inst. H. Poincaré Anal. Non Linéaire., 1 (1984), 223-283.

[19] W.M. Ni and I. Takagi, On the shape of least-energy solutions to a semilinear Neumann problem, Comm. Pure Appl. Math., 44 (1991), 819-851.

[20] C.A. Sackett, H.T.C. Stoof, R.G. Hulet, Growth and collapse of a Bose-Einstein condensate with attractive interactions. Phys. Rev. Lett., 80,(1998), 2031.

[21] M. I. Weinstein, Nonlinear Schrödinger equations and sharp interpolation estimates, Comm. Math. Phys., 87,(1983), 567-576.

[22] M. Willem, Minimax Theorems, Birkhauser, Boston, Basel, Berlin, 1996.

[23] Jianfu Yang and Jinge Yang, Existence and mass concentration of pseudo-relativistic Hartree equation, Journal of Mathematical Physics 58, 081501 (2017); doi: 10.1063/1.4996576 